\title{
Construction of solutions to the $L^{2}$-critical $\mathrm{KdV}$ equation with a given asymptotic behaviour
}

\author{
Raphaël Côte
}

\begin{abstract}
We consider the critical Korteweg-de Vries equation :

$$
u_{t}+\left(u_{x x}+u^{5}\right)_{x}=0, \quad t, x \in \mathbb{R} .
$$

Let $R_{j}(t, x)=Q_{c_{j}}\left(x-x_{j}-c_{j} t\right)(j=1, \ldots, N)$ be $N$ soliton solutions to this equation. Denote $U(t)$ the KdV linear group, and let $V \in H^{1}$ be with sufficient decay on the right, that is $\left(1+x_{+}^{2+\delta_{0}}\right) V \in L^{2}$ for some $\delta_{0}>0$.

We construct a solution $u(t)$ to the critical Korteweg-de Vries equation such that

$$
\lim _{t \rightarrow \infty}\left\|u(t)-U(t) V-\sum_{j=1}^{N} R_{j}(t)\right\|_{H^{1}}=0 .
$$
\end{abstract}

\section{Introduction.}

\subsection{General setting}

We consider the critical Korteweg-de Vries equation :

$$
u_{t}+\left(u_{x x}+u^{5}\right)_{x}=0, \quad t, x \in \mathbb{R} .
$$

It is a special case of the generalized $\mathrm{KdV}$ equation :

$$
u_{t}+\left(u_{x x}+u^{p}\right)_{x}=0, \quad t, x \in \mathbb{R},
$$

where $p \geq 2$. The case $p=2$ corresponds to the original equation introduced by Korteweg and de Vries [8] in the context of shallow water waves. For both $p=2$ and $p=3$, this equation has many applications to Physics : see for example Miura [20], Lamb [10].

There are two formally conserved quantities for solutions to (2) :

$$
\begin{gathered}
\int u^{2}(t)=\int u^{2}(0) \quad\left(L^{2} \text { mass }\right), \\
E(u(t))=\frac{1}{2} \int u_{x}^{2}(t)-\frac{1}{p+1} \int u^{p+1}(t)=E(u(0)) \quad \text { (energy). }
\end{gathered}
$$

The local Cauchy problem for (2) has been intensively studied by many authors. Kenig, Ponce and Vega [6] proved the following existence and uniqueness result

2000 Mathematical Subject Classification : Primary 35Q53, Secondary 35B40, 35Q51.

Keywords : Critical and Generalized Korteweg-de Vries equations, wave operator, large data, solitons, linear-non linear interaction. 
in $H^{1}(\mathbb{R})$ : for $u_{0} \in H^{1}(\mathbb{R})$, there exist $T=T\left(\left\|u_{0}\right\|_{H^{1}}\right)>0$ and a solution $u \in C\left([0, T], H^{1}(\mathbb{R})\right)$ to $(2)$ satisfying $u(0)=u_{0}$, which is unique in the class $Y_{T} \subset C\left([0, T], H^{1}(\mathbb{R})\right)$. Moreover, if $T_{1}$ denotes the maximal time of existence for $u$, then either $T_{1}=+\infty$ (global solution) or $T_{1}<\infty$ and $\|u(t)\|_{H^{1}} \rightarrow \infty$ as $t \uparrow T_{1}$ (blow-up solution). For such a solution, one has conservation of mass and energy. In the critical case, problem 1), this result is improved to local well-posedness in $L^{2}$ (see [6] and [7]).

The next problem is to know whether these solutions to (2) are global in time, or blow-up. In the case $2 \leq p<5$ (sub-critical), all solutions in $H^{1}$ are global and uniformly bounded thanks to the conservation laws and the Gagliardo-Nirenberg inequality :

$$
\forall v \in H^{1}(\mathbb{R}), \quad \int|v|^{p+1} \leq \kappa(p)\left(\int v^{2}\right)^{\frac{p+3}{4}}\left(\int v_{x}^{2}\right)^{\frac{p-1}{4}} .
$$

The case $p=5$ is $L^{2}$-critical, in the sense that mass remains unaffected by scaling. Indeed, $u_{\lambda}(t, x)=\lambda^{1 / 6} u\left(\lambda t, \lambda^{1 / 3} x\right)$ is also a solution to (1), and $\left\|u_{\lambda}\right\|_{L^{2}}=$ $\|u\|_{L^{2}}$. Moreover, existence of finite time blow-up solutions was proved by Merle [19] and Martel and Merle [15]. Therefore $p=5$ also appears as a critical exponent for the long time behaviour of solution to (2).

For $p>5$ (super-critical case), numerics predict blow-up.

A fundamental property of (2) is the existence of a family of explicit traveling wave solutions. If $Q$ denotes the only solution (up to translation) of :

$$
Q>0, \quad Q \in H^{1}(\mathbb{R}), \quad Q_{x x}+Q^{p}=Q, \quad \text { i.e. } \quad Q(x)=\left(\frac{3}{\cosh ^{2}(2 x)}\right)^{1 /(p-1)}
$$

then for $c>0$ the soliton

$$
R_{c, x_{0}}=c^{1 /(p-1)} Q\left(\sqrt{c}\left(x-x_{0}-c t\right)\right) \text { is a solution to }(2) \text {. }
$$

Solitons are stable in $H^{1}$ in the sub-critical case $p \in[2,5)$ (see [13], and unstable in the $p>5$ super-critical (see [22]) and $p=5$ critical case (see [14]).

For $p=2$ and $p=3$, equation (2) is completely integrable, and thus has very special features. The inverse scattering transform method allows to solve the Cauchy problem in an appropriate space (for example if $u_{0} \in H^{4}$ and $x u_{0} \in L^{1}$ ) and the qualitative behaviour of solutions is well understood. For example, given $u_{0}$ smooth and with rapid decay, there exist $N$ solitons $R_{c_{j}, x_{j}}$ such that

$$
\left\|u(t)-\sum_{j=1}^{N} R_{c_{j}, x_{j}}(t)\right\|_{L^{\infty}\left(x \geq-t^{1 / 3}\right)} \leq \frac{C}{t^{1 / 3}} \quad(\text { as } t \rightarrow \infty) .
$$

See for example Schuur [21], Eckhaus and Schuur [4], Miura [20].

However, if $p \neq 2$ or 3 , the inverse scattering transform method does not longer apply, and the description of solutions in the general, non-integrable case is a widely open problem, especially in the critical case. It can be decomposed in two types of problems.

Problem 1: Asymptotic behavior. In the sub-critical case, given an initial data $u_{0}$, can we describe the behavior of the out-coming solution $u(t)$ to (2) ? In the 
critical and super-critical cases, does $u(t)$ blow-up ? Can we determine the blow up rate and profile?

Problem 2: Construction of a non-linear wave operator. Given some reasonable behavior at $t \rightarrow \infty$, can we find a solution $u(t)$ to (2) defined for large enough $t$, with this behavior? Is there uniqueness for $u(t)$ ?

\subsection{Recent results on Problems 1. and 2. for the critical $\mathrm{KdV}$ equation}

From now on, we will focus only on equation (1), that is, the $L^{2}$-critical case.

Let us now develop some results which will be the base to our result. The first result deals with scattering for small initial data. One wants to prove that given an initial data $u_{0}$, small in an adequate functional space, the arising solution to the non-linear equation (1) behaves likes $U(t) v_{0}$, the solution to the linear equation $u_{t}+u_{x x x}=0$, with initial data $v_{0}(U(t)$ is the linear $\mathrm{KdV}$ group). The map $u_{0} \mapsto v_{0}$ is called the scattering operator. The following result is an easy corollary of Kenig, Ponce and Vega [6].

Scattering operator. Given $u_{0}$ small enough in $L^{2}$, the out-coming solution $u(t)$ to (1) is global in time, and there is scattering, in the sense that there exists a function $V \in L^{2}$ so that

$$
\|u(t)-U(t) V\|_{L^{2}} \rightarrow 0 \quad \text { as } \quad t \rightarrow \infty .
$$

This is the description of solutions with initial data around 0 (in $L^{2}$ ), a result which can be understood as stability around 0 .

The second point answers the question of behavior of solutions with initial data close to a soliton. As we are in the critical case, one does not have stability: contrary to the sub-critical case (see [14]), one has instability and blow-up. Let us cite a result of Merle [19] and Martel and Merle [16].

Blow-up solutions to (1). There exists $\alpha_{0}>0$ such that the following is true. Suppose

$$
E(u)<0 \quad \text { and } \quad \int u(t)^{2} \leq \int Q^{2}+\alpha_{0} .
$$

Then $u(t)$ blows-up in finite or infinite time $T \in(0, \infty]$. Furthermore, there exist $\lambda(t)>C(T-t)^{-1 / 3}, \varepsilon \in\{-1,1\}$ and $x(t) \in \mathbb{R}$ such that

$$
\varepsilon \lambda^{1 / 2}(t) u(t, \lambda(t) x+x(t)) \rightarrow Q \quad \text { in } \quad H^{1} \text {-weak } \quad \text { as } \quad t \uparrow T .
$$

These results are related to Problem 1. Let us now turn to results concerning Problem 2. A surprising result of Martel [11] is the existence and uniqueness of $N$-solitons in the critical case :

Existence and uniqueness of the $N$-soliton. Let $p \in[2,5]$. Let $N \in \mathbb{N}, 0<$ $c_{1}<\ldots<c_{N}$, and $x_{1}, \ldots, x_{N} \in \mathbb{R}$. There exist $T_{0} \in \mathbb{R}$ and a unique function $u \in C\left(\left[T_{0},+\infty\right), \mathbb{R}\right)$, which is a $H^{1}$ solution to (2), and such that

$$
\left\|u(t)-\sum_{j=1}^{N} Q_{c_{j}}\left(\cdot-x_{j}-c_{j} t\right)\right\|_{H^{1}} \rightarrow 0 \quad \text { as } \quad t \rightarrow \infty .
$$


Furthermore, $u \in C^{\infty}\left(\left[T_{0}, \infty\right) \times \mathbb{R}\right)$ and convergence takes place in $H^{s}$ for all $s \geq 0$, with an exponential decay:

$$
\forall s \geq 0, \exists A_{s} /\left\|u(t)-\sum_{j=1}^{N} Q_{c_{j}}\left(\cdot-x_{j}-c_{j} t\right)\right\|_{H^{1}} \leq A_{s} e^{-\gamma t},
$$

where $\gamma=\sigma_{0} \sqrt{\sigma_{0}} / 32$ and $\sigma_{0}=\min \left(c_{1}, c_{2}-c_{1}, \ldots, c_{N}-c_{N-1}\right)$.

This result appears as a development of monotonicity properties and a dynamical argument, ideas which where used by Martel and Merle [13] and Martel, Merle and Tsai [17]. It is a surprise that the argument applies also in the critical case $p=5$, although it fails in the proof of stability (failure which isn't due to a lack in the proof, but to true instability : see [14], [16]). The second surprise is the uniqueness of a solution behaving as a sum of $N$ solitons.

The last result solves the case of a linear behavior, that is the existence of a wave operator (see [3]).

Large data wave operator. Let $V \in L^{2}$. There exist $T_{0} \in \mathbb{R}$ and a function $u \in C\left(\left[T_{0}, \infty\right), L^{2}\right)$ solution to (1) such that

$$
\|u(t)-U(t) V\|_{L^{2}} \rightarrow 0 \quad \text { as } \quad t \rightarrow \infty .
$$

Furthermore $u$ is unique in an adapted class.

In the same way that the result of Martel [11] was based on considerations of Martel, Merle and Tsai [17], this result relies on the analysis of Kenig, Ponce and Vega [6].

\subsection{Statement of the main result}

Our goal in this article is to construct solutions which behave like a sum of a linear term $U(t) V$, and of $N$ solitons, for the $L^{2}$-critical Korteweg-de Vries equation (1). Our main result is the following.

Theorem 1 (Nonlinear wave operator for (1)). Let $V \in H^{1}$ have sufficient decay on the right, i.e. such that $\left(1+x_{+}\right)^{2+\delta_{0}} V(x) \in L^{2}$ for some $\delta_{0}>0$ (we denote $\left.x_{+}=\max \{0, x\}\right)$.

Let $N \in \mathbb{N}, 0<c_{1}<\ldots<c_{N}$ and $x_{1}, \ldots, x_{N} \in \mathbb{R}$. Let $R_{j}(t, x)=Q_{c_{j}}(x-$ $\left.x_{j}-c_{j} t\right)$, for $j=1, \ldots, N$, be $N$ solitons.

Then there exists $u^{*} \in C\left(\left[T_{0},+\infty\right), H^{1}\right)$, for some $T_{0} \in \mathbb{R}$, solution to (1) and such that $u^{*}(t)$ is uniformly bounded in $H^{1}$ and

$$
\left\|u^{*}(t)-U(t) V-\sum_{j=1}^{N} R_{j}(t)\right\|_{H^{1}} \rightarrow 0 \quad \text { as } \quad t \rightarrow \infty
$$

Theorem 1 allows to work with large data ( $V$ large in $L^{2}$ ), which is both surprising and satisfactory. The decay on the right we assume for $V$ is to ensure low interaction with the solitons. This result should be viewed as a step in the solving process of Problem 2. 
Remark 1. This result essentially units the linear approach contained in [7] and [3], and the solitons related approach, developed in [18] and [11]. The difficulty is to mix both methods together, so that they do not break down.

An important change in the method of proof when considering [11] is the following. Solitons have an exponential decay, and so integrability (in time) is always automatic. Here the linear term $U(t) V$ will interact with the solitons to produce a polynomial decay in time, which will require to be taken care of.

Remark 2. This result is analogous to that obtained in [2], where a non-linear wave operator is constructed in the sub-critical case $p=4$. However, in the sub-critical case, much more decay and smoothness are required on $V$. This is due to the fact that the linear scattering analysis of [7] is no longer available if $p \neq 5$.

In the sub-critical case,we have to relie on the scattering theory of Hayashi and Naumkin [5]. There it is proved scattering for small data $u_{0} \in H^{1,1}=\{u \in$ $\left.H^{1} \mid x u \in H^{1}\right\}$ : for $p>3$, given such a $u_{0}$ the out-coming solution $u(t)$ to $(2)$ is global, satisfies the linear decay rate $\|u(t)\|_{L^{\infty}} \leq C t^{-1 / 3}$, and there exists $V \in L^{2}$ such that $\|u(t)-U(t) V\|_{L^{2}} \rightarrow 0$ as $t \rightarrow \infty$. Their method is a very beautiful clock-work, but breaks down at some point when constructing the non-linear wave operator. To recover from this, the setting must be strengthen, and hence, the conditions on $V$ must be reinforced.

Here, the methods of [11] and [7] can be smoothly adapted to take care of the interaction between non-linear terms (the solitons) and the linear term $(U(t) V)$, to provide an almost sharp result.

Indeed, our smoothness assumption $H^{1}$ is a natural setting to work with the solitons, and especially to have bounded energy. On the other side, notice that the decay assumption only concerns the $L^{2}$ level for $V$, and only decay on the right. The assumption on $U(t) V$ should be understood in this way : to handle the interaction of the solitons, we need one degree of decay on $V$ so that its interference is low enough. To prevent the solitons from interfering too much when handling the linear term $U(t) V$, we need a second order of decay on $V$.

An optimal result for our framework would then be $\left(1+x_{+}^{2}\right) V(x) \in L^{2}(d x)$. In view of this, our assumption appears to be almost optimal.

Remark 3. The uniqueness of solutions to (1) with a given asymptotic behaviour of the form $U(t) V+R(t)$ is not clear. Remind that for $V=0$, that is, the $N$-soliton, one has uniqueness in $H^{1}$ (see [11]) : it is linked to the fact the constructed solution is smooth and converges exponentially fast in $H^{s}$ for all $s \geq 0$ ( $s=4$ would be enough). If $V$ belong to $H^{1}$ but not more, this is not possible. However, one might be able to prove uniqueness for smoother $V$.

Remark 4. There are some analogous results for the (critical) non-linear Schrödinger equation. See Bourgain and Wang [1], Krieger and Schlag [9], Merle [18]. In [1], a solution to the critical NLS equation with a given blow-up behaviour is constructed : thanks to the conformal transform, this is in fact equivalent to construct a solution to the critical NLS equation which behaves like the sum of a soliton and a linear term. High smoothness and low interaction with the soliton are required on the linear term.

\section{Acknowledgment}


I would like to thank my advisor Frank Merle for numerous discussions, Luis Vega for his invitation, and Herbert Koch for bringing my attention to similar questions. This research was supported in part by the European network HYKE as contract HPRN-CT-2002-00282 and by the University of the Basque Country.

\section{Strategy of the proof.}

Following a usual convention, different positive constants might be denoted by the same letter $C$.

Let $V$ as in the hypothesis of Theorem $1,0<c_{1}<\ldots<c_{N}$ and $x_{1}, \ldots, x_{N} \in$ $\mathbb{R}$. Denote the soliton with speed $c_{j}$ and shift $x_{j}$

$$
R_{j}(t, x)=Q_{c_{j}}\left(x-x_{j}-c_{j} t\right)
$$

Define also $R(t)=\sum_{j=1}^{N} R_{j}(t)$.

Let $S_{n}$ be an increasing sequence of time, so that $S_{n} \rightarrow \infty$ as $n \rightarrow \infty$ and

$$
\lim _{n \rightarrow \infty} E\left(U\left(S_{n}\right)+R\left(S_{n}\right)\right)=\liminf _{t \rightarrow \infty} E\left(U\left(S_{n}\right)+R\left(S_{n}\right)\right) .
$$

(such a sequence obviously exists ; the condition on the energy appears when concluding the proof of Theorem 1$)$. For $n>0$, we define $u_{n}(t)$, the solution of

$$
\left\{\begin{array}{l}
u_{n t}+\left(u_{n x x}+u^{5}\right)_{x}=0 \\
u_{n}\left(S_{n}\right)=U\left(S_{n}\right)+R\left(S_{n}\right) .
\end{array}\right.
$$

Equivalently, we introduce $w_{n}(t)$ the error term

$$
w_{n}(t)=u_{n}(t)-U(t) V-R(t),
$$

so that $w_{n}(t)$ satisfies the equation

$$
\left\{\begin{array}{l}
w_{n t}+w_{n x x x}+\left(u^{5}-\sum_{j=1}^{N} R_{j}^{5}(t)\right)_{x}=0, \\
w_{n}\left(S_{n}\right)=0 .
\end{array}\right.
$$

As $u\left(S_{n}\right) \in H^{1}, u_{n}(t)$ is well defined, at least on a small interval of time around $S_{n}$.

The heart of the proof of Theorem 1 is the following result :

Proposition 1 (Uniform estimates). There exist $T_{0}, K_{0}$ and a continuous function $\eta:[1, \infty) \rightarrow \mathbb{R}_{*}^{+}$, depending on $V$, with

$$
\eta(t) \downarrow 0 \quad \text { as } \quad t \rightarrow \infty,
$$

such that the following is true. For all $n$ such that $S_{n} \geq T_{0}$, the solution $u_{n}(t)$ to (8) and the solution $w_{n}(t)$ to $(9)$ belong to $C\left(\left[T_{0}, S_{n}\right], H^{1}\right)$. Furthermore, we have the uniform decay estimate and control (in $n$ ):

$$
\forall t \in\left[T_{0}, S_{n}\right], \quad\left\|w_{n}(t)\right\|_{L^{2}} \leq \eta(t), \quad \text { and } \quad\left\|w_{n}(t)\right\|_{H^{1}} \leq K_{0}
$$

The proof of this proposition requires several steps.

The first remark allows us to further assume smallness on $w_{n}(t)$, in order to get the decay (10). 
Proposition 1' (Reduction of proof). There exist $\varepsilon_{0}>0, T_{0} \geq 1$ and a decreasing continuous function $\eta:[1, \infty) \rightarrow \mathbb{R}_{*}^{+}$, depending on $V$, with

$$
\eta(t) \downarrow 0 \quad \text { as } t \rightarrow \infty,
$$

such that the following is true. Introduce the norm

$$
\|f(t, x)\|_{\mathcal{N}([A, B])}=\|f\|_{L_{x}^{5} L_{t}^{10}(t \in[A, B])}+\sup _{t \in[A, B]}\|f(t)\|_{L_{x}^{2}} .
$$

Let $n \in \mathbb{N}$ so that $S_{n} \geq T_{0}$. Let $I_{n} \in\left[T_{0}, S_{n}\right]$ such that $\left\|w_{n}\right\|_{\mathcal{N}\left(\left[I_{n}, S_{n}\right]\right)} \leq \varepsilon_{0}$. Then in fact,

$$
\forall t \in\left[I_{n}, S_{n}\right], \quad\left\|w_{n}\right\|_{\mathcal{N}\left(\left[t, S_{n}\right]\right)} \leq \eta(t), \quad \text { and } \quad\left\|w_{n}(t)\right\|_{H^{1}} \leq K_{0} .
$$

We introduce the $L_{x}^{5} L_{t}^{10}$ space as it is necessary in the control of the linear term $U(t) V$ : see [7] for further details.

Proof of Proposition 1 assuming Proposition 1'. This is a continuity argument. Let

$$
T_{0}=\inf \left\{\tau: \tau \geq 1 \text { and } \eta(\tau) \leq \varepsilon_{0}\right\}
$$

and define

$$
I_{n}^{*}=\inf \left\{\tau: \tau \in\left[1, S_{n}\right], \text { and }\left\|w_{n}\right\|_{\mathcal{N}\left(\left[\tau, S_{n}\right]\right)} \leq \varepsilon_{0}\right\}
$$

We now use the continuity the norm $L_{x}^{5} L_{t}^{10} \cap C^{0} H^{1}$ under the flow of (1), (see [7]). As $w_{n}\left(S_{n}\right)=0$, we obtain that the set on which we do the infimum is non-empty, so that $I_{n}^{*}<S_{n}$.

Then of course, this allows us to apply Proposition 1' with $I_{n}=I_{n}^{*}$ so that

$$
\forall t \in\left[I_{n}^{*}, S_{n}\right], \quad\left\|w_{n}\right\|_{\mathcal{N}\left(\left[t, S_{n}\right]\right)} \leq \eta(t), \quad \text { and } \quad\left\|w_{n}(t)\right\|_{H^{1}} \leq K_{0}
$$

By minimality of $I_{n}^{*}$, if $I_{n}^{*}>1$, we also get that

$$
\limsup _{t \downarrow I_{n}^{*}}\left\|w_{n}\right\|_{\mathcal{N}\left(\left[t, S_{n}\right]\right)} \geq \varepsilon_{0} .
$$

In particular, this gives

$$
\varepsilon_{0} \leq \limsup _{t \downarrow I_{n}^{*}}\left\|w_{n}\right\|_{\mathcal{N}\left(\left[t, S_{n}\right]\right)} \leq \limsup _{t \downarrow I_{n}^{*}} \eta(t) \leq \eta\left(I_{n}^{*}\right) .
$$

So that $\eta\left(I_{n}^{*}\right) \geq \varepsilon_{0}$.

In any case, we get that $I_{n}^{*} \leq T_{0}$ (as $\eta$ is decreasing) : (11) allows us to conclude.

Proof of Proposition 1'.

Step 1 : Monotonicity and non-linear tools. We obtain $L^{2}$ estimates on the right. Let us introduce the cut-off speed

$$
\sigma_{0} \in\left(0,1 / 2 \min \left\{c_{1}, c_{2}-c_{1}, \ldots, c_{N}-c_{N-1}\right\}\right),
$$

to be determined in the proof of the following Proposition 2 below, and the cut-off function

$$
\psi(x)=\frac{2}{\pi} \arctan \left(\exp \left(-\frac{\sqrt{\sigma_{0}}}{2} x\right)\right), \quad \psi_{0}(t, x)=\psi\left(x-\sigma_{0} t-2\left|x_{1}\right|\right) .
$$

$\psi_{0}(t)$ allows us to separate the solitons interaction from the $U(t) V$ interaction. 
Proposition 2 (Interaction with the solitons). There exist $\sigma_{1}>0, \varepsilon_{1}, T_{1}$, $C_{1}$ and $K_{0}$ such that the following is true. If $\sigma_{0} \leq \sigma_{1}, \varepsilon_{0} \leq \varepsilon_{1}$ and $T_{0} \geq T_{1}$, then, for all $n \in \mathbb{N}$ and all $t \in\left[I_{n}, S_{n}\right]$,

$$
\begin{aligned}
& \|w(t)\|_{L^{2}\left(1-\psi_{0}(t)\right)} \leq C_{1} e^{-\frac{\sigma_{0} \sqrt{\sigma_{0}}}{8} t}+C_{1}\|U(t) V\|_{L^{2}\left(1-\psi_{0}(t)\right)} \\
& \quad+C_{1}\left(S_{n}-t+1\right)\|U(t) V\|_{L^{2}\left(1-\psi_{0}\left(S_{n}\right)\right)}+C_{1} \int_{t}^{S_{n}}\|U(t) V\|_{L^{2}\left(1-\psi_{0}(t)\right)} d t,
\end{aligned}
$$

and

$$
\left\|w_{n}(t)\right\|_{H^{1}} \leq K_{0} .
$$

The control of the $H^{1}$-norm simply relies on uniform bounds of the energy, and on the smallness assumption on $\|w(t)\|_{L^{2}}$. The deep result is the first estimate.

Essentially we obtain a polynomial decay on $\left\|w_{n}(t)\right\|_{L^{2}\left(1-\psi_{0}(t)\right)}$ (instead of an exponential decay in the case of solely soliton). However the good point is that we can choose this polynomial decay to be as fast as we want by lowering the interaction of $U(t) V$ with the solitons, that is, by requiring sufficient decay on the right for $V$ : see Lemma 2 .

Step 2: Linear theory. Essentially we have to take care of the interaction of $U(t) V$ and $w_{n}$. For this, we use the linear estimates and the setting of [6] and [7].

Proposition 3 (Ineteraction with the linear term). There exists $\varepsilon_{2}>0$, $T_{2}, C_{2}$ such that the following is true. Suppose that for some $C$ and $\delta_{0}>0$, we have for all $n$ such that $S_{n} \geq T_{2}$ :

$$
\forall t \in\left[I_{n}, S_{n}\right], \quad\left\|w_{n}(t)+U(t) V\right\|_{L^{2}\left(1-\psi_{0}(t)\right.} \leq \frac{C}{t^{1+\delta_{0}}} .
$$

Then there exists $C_{2}$ such that if we denote:

$$
\eta(t)=C_{2}\|U(\tau) V\|_{L_{x}^{5} L_{\tau}^{10}(\tau \geq t)}^{5}+C_{2} e^{-\frac{\sigma_{0} \sqrt{\sigma 0}}{4} t}\|U(\tau) V\|_{L_{x}^{5} L_{\tau}^{10}(\tau \geq t)}+\frac{C_{2}}{t^{\delta_{0}}},
$$

we have :

$$
\left\|w_{n}(t)\right\|_{\mathcal{N}\left(\left[I_{n}, S_{n}\right]\right)} \leq \eta(t) .
$$

Of course, $\eta(t)$ decreases to 0 as $t \rightarrow \infty$, and so satisfies the conditions of Proposition 1'.

Finally, Proposition 2, Lemma 2 and estimates (42) and (43) ensure that the assumptions of Proposition 3 are fulfilled if $V$ is chosen as in Theorem 1, that is $V \in H^{1}$ and $x_{+}^{2+\delta_{0}} V \in L^{2}$. Fix $\sigma_{0}<\sigma_{1}, \varepsilon_{0}=\min \left\{\varepsilon_{1}, \varepsilon_{2}\right\}$ and $T_{0}=\min \left\{T_{1}, T_{2}\right\}:$ this completes the proof of Proposition 1', and so, of Proposition 1.

Proof of Theorem 1. From Proposition 1, we are able to prove some compactness property in $L^{2}$ on the sequence $u_{n}\left(T_{0}\right)$. The limit of a subsequence yields an initial data $\varphi_{0}$, from which $u^{*}(t)$ is the out-coming solution to (1). Then Proposition 1 allows to conclude that

$$
\left\|u^{*}(t)-U(t) V-R(t)\right\|_{L^{2}} \rightarrow 0 .
$$


To obtain the $H^{1}$ convergence, we need another argument. We compare $E\left(U\left(S_{n}\right)+R\left(S_{n}\right)\right)$ and $E\left(u^{*}(t)\right)$, taking advantage of (7). By developping

$$
E\left(u^{*}(t)\right)=E\left(w^{*}(t)+U(t) V+R(t)\right)
$$

and studying carefully all the obtained terms, we finally prove that the error term $\left\|w_{x}^{*}(t)\right\|_{L^{2}} \rightarrow 0$ as $t \rightarrow \infty$ : this completes the proof of Theorem 1 .

The proof of Theorem 1 assuming Proposition 1 is done in Section 3. The rest of the proof completes the proof of Proposition 1' and thus that of Proposition 1. In Section 4., we give some preliminary estimates to be used both in Section 5. and Section 6. Section 5. is devoted the proof of Proposition 2. Finally, Proposition 3 is proved in Section 6.

\section{Proof of Theorem 1 assuming Proposition 1}

In this section, we assume Proposition 1 holds, and from this we conclude the proof of Theorem 1.

\subsection{A compactness result linked with the monotonicity Lemma 5}

From Proposition 1, we dispose of a sequence $u_{n}(t)$ defined on $\left[T_{0}, S_{n}\right]$ (we dropped the terms with $S_{n}<T_{0}$ ), solutions to (2), such that

$$
u_{n}\left(S_{n}\right)=U\left(S_{n}\right) V+\sum_{j=1}^{N} R_{j}\left(S_{n}\right)=U\left(S_{n}\right)+R\left(S_{n}\right),
$$

and that the following uniform estimates holds $\left(w_{n}(t)=u_{n}(t)-U(t) V-R(t)\right)$ :

$$
\forall n \in \mathbb{N}, \forall t \in\left[T_{0}, S_{n}\right], \quad\left\|w_{n}(t)\right\|_{L^{2}} \leq \eta(t) \quad \text { and } \quad\|w(t)\|_{H^{1}} \leq K_{0} .
$$

Claim. $u_{n}\left(T_{0}\right)$ is a compact sequence in the sense that

$$
\lim _{A \rightarrow \infty} \sup _{n \in \mathbb{N}} \int_{|x| \geq A} u_{n}{ }^{2}\left(T_{0}, x\right) d x=0 .
$$

Proof of the Claim. Indeed, let $\varepsilon>0$, and $T(\varepsilon)$ such that $\eta(T(\varepsilon)) \leq \sqrt{\varepsilon}$. Then

$$
\int\left(u_{n}(T(\varepsilon))-U(T(\varepsilon)) V-R(T(\varepsilon))^{2} \leq \varepsilon .\right.
$$

Let $A(\varepsilon)$ be such that $\int_{|x| \geq A(\varepsilon)}(U(T(\varepsilon)) V+R(T(\varepsilon)))^{2}(x) d x \leq \varepsilon$; we get

$$
\int_{|x| \geq A(\varepsilon)} u_{n}^{2}(T(\varepsilon), x) d x \leq 2 \varepsilon .
$$

Let $g \in C^{3}$ a function such that $g(x)=0$ if $x \leq 0, g(x)=1$ if $x \geq 2$, and furthermore $0 \leq g^{\prime}(x) \leq 1,0 \leq g^{\prime \prime \prime}(x) \leq 1$.

Remind that if $f \in C^{3}$ does only depend on $x$, we have

$$
\frac{d}{d t} \int u_{n}^{2} f=-3 \int u_{n x}^{2} f_{x}+\int u_{n}^{2} f_{x x x}+\frac{2 p}{p+1} \int u_{n}^{p+1} f_{x} .
$$


(See Lemma 5 and its proof). For $C(\varepsilon)$ to be determined later, we then have

$$
\begin{aligned}
& \frac{d}{d t} \int u_{n}{ }^{2}(t, x) g\left(\frac{x-A(\varepsilon)}{C(\varepsilon)}\right)=-\frac{3}{C(\varepsilon)} \int u_{n_{x}}^{2} g^{\prime}\left(\frac{x-A(\varepsilon)}{C(\varepsilon)}\right) \\
& \quad+\frac{1}{C(\varepsilon)^{3}} \int u_{n}{ }^{2} g^{\prime \prime \prime}\left(\frac{x-A(\varepsilon)}{C(\varepsilon)}\right)+\frac{2 p}{(p+1) C(\varepsilon)} \int u_{n}{ }^{p+1} g^{\prime}\left(\frac{x-A(\varepsilon)}{C(\varepsilon)}\right) .
\end{aligned}
$$

As $t \geq T_{0}, u_{n}$ satisfies $\left\|u_{n}(t)\right\|_{H^{1}} \leq K_{0}+\|V\|_{H^{1}}+\sum_{j=1}^{N}\left\|Q_{c_{j}}\right\|_{H^{1}} \leq C^{0}$. So that :

$$
\begin{aligned}
\mid \frac{d}{d t} \int u_{n}{ }^{2}(t & x) g\left(\frac{x-A(\varepsilon)}{C(\varepsilon)}\right) \mid \\
& \leq \frac{1}{C(\varepsilon)}\left(3 \int u_{n_{x}}^{2}(t)+\int u_{n}{ }^{2}(t)+\frac{2 p}{p+1}\left\|u_{n}\right\|_{L^{\infty}}^{p-1} \int u_{n}{ }^{2}(t)\right) \\
& \leq \frac{1}{C(\varepsilon)}\left(3 C^{0^{2}}+\frac{2 p}{p+1} 2^{(p-1) / 2} C^{0 p+1}\right) .
\end{aligned}
$$

Now choose $C(\varepsilon)=\max \left\{1, \frac{T(\varepsilon)-T_{0}}{\varepsilon}\left(3 C^{0^{2}}+\frac{2 p}{p+1} 2^{(p-1) / 2} C^{0 p+1}\right)\right\}$, from which we derive

$$
\left|\frac{d}{d t} \int u_{n}{ }^{2}(t, x) g\left(\frac{x-A(\varepsilon)}{C(\varepsilon)}\right)\right| \leq \frac{\varepsilon}{T(\varepsilon)-T_{0}} .
$$

And after integration in time between $T_{0}$ and $T(\varepsilon)$,

$$
\int_{x \geq 2 C(\varepsilon)+A(\varepsilon)} u_{n}{ }^{2}\left(T_{0}, x\right) \leq \int u_{n}{ }^{2}\left(T_{0}, x\right) g\left(\frac{x-A(\varepsilon)}{C(\varepsilon)}\right) \leq 3 \varepsilon .
$$

Now considering $\frac{d}{d t} \int{u_{n}}^{2}(t, x) g\left(\frac{-A(\varepsilon)-x}{C(\varepsilon)}\right)$, we get in a similar way

$$
\int_{x \leq-2 C(\varepsilon)-A(\varepsilon)} u_{n}^{2}\left(T_{0}, x\right) \leq 3 \varepsilon
$$

So that if we denote $A_{\varepsilon}=2 C(\varepsilon / 6)+A(\varepsilon / 6)$, we obtain

$$
\forall n \in \mathbb{N}, \quad \int_{|x| \geq A_{\varepsilon}} u_{n}^{2}\left(T_{0}, x\right) \leq \varepsilon,
$$

as claimed.

\subsection{Construction of $u^{*}$ and $L^{2}$ convergence to the profile}

Now $u_{n}\left(T_{0}\right)$ is a bounded sequence in $H^{1}(\mathbb{R})$, and so converges weakly up to a subsequence, to some $\varphi_{0}$ in $H^{1}(\mathbb{R})$ (we suppose for convenience that the whole sequence converges weakly). The previous compactness result ensures that the convergence is strong in $L^{2}(\mathbb{R})$. Indeed, let $\varepsilon>0$, and $A$ such that $\int_{|x| \geq A} \varphi_{0}^{2}(x) d x \leq \varepsilon$ and

$$
\forall n \in \mathbb{N}, \quad \int_{|x| \geq A} u_{n}^{2}\left(T_{0}, x\right) \leq \varepsilon
$$


The injection $H^{1}([-A, A]) \rightarrow L^{2}([-A, A])$ is compact, so that $\int_{|x| \leq A} \mid u_{n}\left(T_{0}, x\right)-$ $\left.\varphi_{0}(x)\right|^{2} d x \rightarrow 0$. We thus derive that

$$
\limsup _{n \rightarrow \infty}\left\|u_{n}\left(T_{0}\right)-\varphi_{0}\right\|_{L^{2}(\mathbb{R})}^{2} \leq 4 \varepsilon .
$$

As this is true for all $\varepsilon>0, u_{n}\left(T_{0}\right) \rightarrow \varphi_{0}$ in $L^{2}(\mathbb{R})$.

Denote $u^{*}(t)$ the solution to

$$
\left\{\begin{array}{l}
u_{t}^{*}+\left(u_{x x}^{*}+u^{* p}\right)_{x}=0 \\
u^{*}\left(T_{0}\right)=\varphi_{0} .
\end{array}\right.
$$

The Cauchy problem being well-posed in $L^{2}(\mathbb{R}), u^{*}$ is well defined, at least for $t$ in a neighborhood $\mathcal{V}$ of $T_{0}$. Now the flow is continuous in $L^{2}$ (in fact it is Lipschitz), so that for all $t \in \mathcal{V}, u_{n}(t) \rightarrow u^{*}(t)$ in $L^{2}$. As $\left(u_{n}(t)\right)_{n}$ is a bounded sequence in $H^{1}$, this proves that the whole sequence converges weakly to $u^{*}(t)$ in $H^{1}$ :

$\forall t \in \mathcal{V}, \quad \lim _{n \rightarrow \infty} u_{n}(t)=u^{*}(t) \quad$ in $L^{2}(\mathbb{R})$ - strong and $H^{1}(\mathbb{R})$ - weak.

Thus, we can take the limit in the estimates (10) (with $t$ fixed), to get

$\forall t \in \mathcal{V}, \quad\left\|u^{*}(t)-U(t) V-R(t)\right\|_{L^{2}} \leq \eta(t), \quad$ and $\left\|u^{*}(t)-U(t) V-R(t)\right\|_{H^{1}} \leq K_{0}$.

This shows that $u^{*}(t)$ is $H^{1}$ uniformly bounded on $\mathcal{V}$, so that by the Cauchy problem theory and a standard continuity argument, $u^{*}$ is defined for all $t \geq T_{0}$. Hence, $w^{*}(t)$ is uniformly bounded in $H^{1}$, and satisfies the expected $L^{2}$ decay estimate :

$$
\left\|w^{*}(t)\right\|_{H^{1}} \leq K_{0} \quad \text { and } \quad\left\|w^{*}(t)\right\|_{L^{2}} \leq \eta(t) \rightarrow 0 \quad \text { as } \quad t \rightarrow \infty .
$$

\section{3 $H^{1}$ convergence of $u^{*}(t)$ to its profile}

The $H^{1}$ convergence comes essentially from an analysis of the energy $E\left(u^{*}(t)\right)$.

From $(14), u_{n}(t) \rightarrow u^{*}(t) H^{1}$-weak and $u_{n}(t) \rightarrow u^{*}(t)$ in $L^{6}$ as $n \rightarrow \infty$, and we deduce that

$$
\begin{aligned}
E\left(u^{*}\left(T_{0}\right)\right) & \leq \liminf _{n \rightarrow \infty} E\left(u_{n}\left(T_{0}\right)\right) \leq \liminf _{n \rightarrow \infty} E\left(u_{n}\left(S_{n}\right)\right) \\
& \leq \liminf _{n \rightarrow \infty} E\left(U\left(S_{n}\right) V+R\left(S_{n}\right)\right) .
\end{aligned}
$$

Now, conservation of energy gives for $E\left(u^{*}(t)\right)=E\left(u^{*}\left(T_{0}\right)\right)$, for $t \geq T_{0}$. By $(7)$, and in view of the previous computation, we have

$$
\begin{aligned}
\liminf _{t \rightarrow \infty}(E(U(t) & \left.V+R(t))-E\left(u^{*}(t)\right)\right) \\
& =\liminf _{t \rightarrow \infty} E(U(t) V+R(t))-E\left(u^{*}\left(T_{0}\right)\right) \\
& =\lim _{n \rightarrow \infty} E\left(U\left(S_{n}\right) V+R\left(S_{n}\right)\right)-E\left(u^{*}\left(T_{0}\right)\right) \\
& \geq 0 .
\end{aligned}
$$

Thus, let us estimate $E(U(t) V+R(t))-E\left(u^{*}(t)\right)$ :

$$
E(U(t) V+R(t))-E\left(u^{*}(t)\right)
$$




$$
\begin{aligned}
=E & (U(t) V+R(t))-E\left(w^{*}(t)+U(t) V+R(t)\right) \\
=E & (U(t) V+R(t))-E\left(w^{*}(t)\right)-E(U(t) V+R(t))-\int w_{x}^{*}(t) U(t) V_{x} \\
& \left.\quad-\int w_{x}^{*}(t) R_{x}(t)\right)+\frac{1}{6} \sum_{k=1}^{5} C_{6}^{k} \int w^{*}(t)^{k}(U(t) V+R(t))^{6-k} \\
=- & \frac{1}{2} \int\left|w_{x}^{*}(t)\right|^{2}-\int w_{x}^{*}(t) U(t) V_{x}-\int w_{x}^{*}(t) R_{x}(t) \\
& +\frac{1}{6} \sum_{k=1}^{6} C_{6}^{k} \int w^{*}(t)^{k}(U(t) V+R(t))^{6-k} .
\end{aligned}
$$

Remind (15) : by interpolation $L^{2}-H^{1}$, we get that for all $p \geq 2,\left\|w^{*}(t)\right\|_{L^{p}} \rightarrow 0$ as $t \rightarrow \infty$.

Let us first control the second line in (17) : for $k=2, \ldots, 6$,

$$
\left|\int w^{*}(t)^{k}(U(t) V+R(t))^{6-k}\right| \leq\left\|w^{*}(t)\right\|_{L^{k}}^{k}\|U(t) V+R(t)\|_{L^{\infty}}^{6-k}=o_{t \rightarrow \infty}(1) .
$$

For $k=1$, we have also

$$
\begin{aligned}
& \left|\int w^{*}(t)(U(t) V+R(t))^{5}\right| \\
& \quad \leq\left\|w^{*}(t)\right\|_{L^{2}}\|U(t) V+R(t)\|_{L^{2}}\|U(t) V+R(t)\|_{L^{\infty}}^{4}=o_{t \rightarrow \infty}(1) .
\end{aligned}
$$

Now,

$$
\left|\int w_{x}^{*}(t) R_{x}(t)\right|=\left|\int w^{*}(t) R_{x x}(t)\right| \leq\left\|w^{*}(t)\right\|_{L^{2}}\|R(t)\|_{H^{2}}=o_{t \rightarrow \infty}(1) .
$$

The last term $\int w_{x}^{*}(t) U(t) V_{x}$ requires a little more attention. Consider the function $U(-t)\left(w^{*}(t)\right)$ : then $\left\|U(-t)\left(w^{*}(t)\right)\right\|_{L^{2}}=\left\|w^{*}(t)\right\|_{L^{2}} \rightarrow 0$ as $t \rightarrow \infty$ and $\left\|U(-t)\left(w^{*}(t)\right)\right\|_{H^{1}}=w^{*}(t) \|_{H^{1}}$ is uniformly bounded in $t$. Hence, the only possible weak limit of $U(-t)\left(w^{*}(t)\right)$ in $H^{1}$ (as $\left.t \rightarrow \infty\right)$ is 0 . This proves that :

$$
\left\{\begin{array}{l}
U(-t)\left(w^{*}(t)\right) \rightarrow 0 \quad \text { in } L^{2}-\text { strong as } t \rightarrow \infty \\
U(-t)\left(w_{x}^{*}(t)\right) \rightarrow 0 \quad \text { in } L^{2}-\text { weak as } t \rightarrow \infty
\end{array}\right.
$$

This proves that

$$
\int w_{x}^{*}(t) U(t) V_{x}=\int U(-t)\left(w_{x}^{*}(t)\right) V_{x}=o_{t \rightarrow \infty}(1) .
$$

We can conclude from (17) that

$$
E(U(t) V+R(t))-E\left(u^{*}(t)\right)=-\frac{1}{2} \int\left|w_{x}^{*}(t)\right|^{2}+o_{t \rightarrow \infty}(1),
$$

and in view of (16),

$$
\begin{aligned}
0 & \leq \liminf _{t \rightarrow \infty}\left(E(U(t) V+R(t))-E\left(u^{*}(t)\right)\right) \\
& \leq \liminf _{t \rightarrow \infty}\left(-\frac{1}{2} \int\left|w_{x}^{*}(t)\right|^{2}+o_{t \rightarrow \infty}(1)\right)
\end{aligned}
$$




$$
\leq-\frac{1}{2} \limsup _{t \rightarrow \infty} \int\left|w_{x}^{*}(t)\right|^{2} .
$$

This proves that $\left\|w_{x}^{*}(t)\right\|_{L^{2}} \rightarrow 0$, and along with (15), we get that

$$
\left\|u^{*}(t)-U(t) V-R(t)\right\|_{H^{1}} \rightarrow 0 \quad \text { as } \quad t \rightarrow \infty .
$$

This concludes the proof of Theorem 1 .

The following is devoted the proof of Proposition 1, or more precisely of Proposition 1'. We will now only work on the interval $\left[I_{n}, S_{n}\right]$.

\section{Preliminaries}

\subsection{Cut-off functions and localized quantities}

We already introduced $\sigma_{0} \in\left(0,1 / 2 \min \left\{c_{1}, c_{2}-c_{1}, \ldots, c_{N}-c_{N-1}\right\}\right)$, and the cut-off function :

$$
\psi(x)=\frac{2}{\pi} \arctan \left(e^{-\frac{\sqrt{\sigma_{0}}}{2} x}\right) .
$$

We can check that $\lim _{+\infty} \psi=0, \lim _{-\infty} \psi=1$, and $\psi$ is decreasing. Furthermore, by direct computations,

$$
\psi^{\prime}(x)=-\frac{\sqrt{\sigma_{0}}}{2 \pi \cosh \left(\frac{\sqrt{\sigma_{0}}}{2} x\right)}, \quad \psi^{\prime \prime \prime}=\frac{\sigma_{0}}{4} \psi^{\prime}(x)\left(1-\frac{2}{\cosh \left(\frac{\sqrt{\sigma_{0}}}{2} x\right)}\right),
$$

and so,

$$
\left|\psi^{\prime \prime \prime}(x)\right| \leq-\frac{\sigma_{0}}{4} \psi^{\prime}(x) .
$$

We introduce, for $j=1, \ldots, N-1$ :

$m_{j}(t)=\frac{c_{j}+c_{j+1}}{2} t+\frac{x_{j}+x_{j+1}}{2}, \quad m_{0}(t)=\sigma_{0} t-2\left|x_{1}\right|, \quad m_{-1}(t)=\frac{\sigma_{0}}{2} t-2\left|x_{1}\right|$.

So that we can define, for $j=-1, \ldots, N-1$ :

$$
\psi_{j}(t, x)=\psi\left(x-m_{j}(t)\right), \quad \psi_{N}(t, x)=1 .
$$

Then we set, for $j=1, \ldots, N-1$ :

$$
\phi_{0}(t)=\psi_{0}(t), \quad \phi_{j}(t)=\psi_{j}(t)-\psi_{j-1}(t), \quad \phi_{N}(t)=1-\psi_{N-1}(t) .
$$

By construction, $\sum_{k=1}^{j} \phi_{k}=\psi_{j}$. Finally, we define some local quantities related to mass and energy :

$$
\begin{aligned}
& M_{j}(t)=\int u_{t}^{2}(t) \phi_{j}(t), \quad E_{j}(t)=\int\left(\frac{1}{2} u_{x}^{2}(t)-\frac{1}{p+1} u^{p+1}(t)\right) \phi_{j}(t), \\
& F_{j}(t)=E_{j}(t)+\frac{1}{100} M_{j}(t) .
\end{aligned}
$$

For $j \geq 1$, the $\phi_{j}$ separates the solitons $R_{j}$ from one another. $\psi_{0}(t)$ separates the solitons from the linear term $U(t) V$. The aim of $\psi_{-1}(t)$ is different : it provides an interval on which $U(t) V$ is small in $H^{1}$ an so in $L^{\infty}$ (see Lemma 2 hereafter). This will be crucially used in the almost monoticity Lemma 5 (it is in fact the only place where $\psi_{-1}(t)$ plays a role).

Observe that $\|U(t) V\|_{L^{\infty}} \leq C\|V\|_{L^{1}} t^{-1 / 3}$, so that pointwise smallness on $U(t) V$ is automatic if $V \in L^{1}$. However, this hypothesis is not part of Theorem 1. 


\subsection{Preliminary bounds on $w_{n}(t)$}

Notice that from the uniform bound on the energy, we get a uniform control on $w_{n}(t)$ for $t \in\left[I_{n}, S_{n}\right]$. This is the purpose of the following lemma. This preliminiary result will be very important in the proof of the almost monotonicity Lemma 5.

Lemma 1 (Bound on the $H^{1}$ norm of $w_{n}(t)$ ). There exists $K_{0}$ independent of $\left.\left.\varepsilon_{0} \in\right] 0, \kappa(6)^{-1 / 4}\right]$ (remind (5)), such that

$$
\forall n \in \mathbb{N}, \forall t \in\left[I_{n}, S_{n}\right], \quad\left\|w_{n}(t)\right\|_{H^{1}} \leq K_{0} .
$$

In particular, the $\|w(t)\|_{L^{\infty}}$ can be made arbitrarily small :

$$
\forall n \in \mathbb{N}, \forall t \in\left[I_{n}, S_{n}\right], \quad\|w(t)\|_{L^{\infty}} \leq \sqrt{\varepsilon_{0} K_{0}} .
$$

Remark that this lemma gives the second estimate of Proposition 1'.

Proof. We combine smallness of $w_{n}(t)$ in $L^{2}$ along with uniform bounds (in $n$ ) on $E\left(u_{n}\right)$. The energy is preserved so that $E\left(u_{n}\left(S_{n}\right)\right)=E\left(u_{n}(t)\right)$. Then we have

$$
\begin{aligned}
& E\left(u_{n}\left(S_{n}\right)\right)=E\left(U\left(S_{n}\right) V+R\left(S_{n}\right)\right) \\
& \leq C \int\left|U\left(S_{n}\right) V_{x}\right|^{2}+C \sum_{j=1}^{N} \int Q_{c_{j} x}^{2}+C \int\left|U\left(S_{n}\right) V\right|^{6}+C \sum_{j=1}^{N} \int Q_{c_{j}}^{6} \leq C .
\end{aligned}
$$

So that the energy $E\left(u_{n}(t)\right)$ is uniformly bounded (in $n$ ). Now we have the following.

Claim. Let $f, \varepsilon \in H^{1}$, with $\|\varepsilon\|_{L^{2}} \leq \kappa(6)^{-1 / 4}$. Then there is a function $G: \mathbb{R}^{+} \rightarrow \mathbb{R}^{+}$such that

$$
\|\varepsilon\|_{H^{1}} \leq 3 E(f+\varepsilon)+G\left(\|f\|_{H^{1}}\right) .
$$

To conclude, it suffice to apply the claim for $\varepsilon=w_{n}(t)$ and $f=U(t) V+R(t)$ (whose $H^{1}$-norm is uniformly bounded in $t$ ).

Let us prove the claim. Indeed, we compute :

$$
\begin{aligned}
E(f+\varepsilon) & =\frac{1}{2} \int(f+\varepsilon)_{x}^{2}-\frac{1}{6} \int(f+\varepsilon)^{6} \\
& =\frac{1}{2} \int f_{x}^{2}+\int f_{x} \varepsilon_{x}+\frac{1}{2} \int \varepsilon_{x}^{2}-\frac{1}{6} \sum_{k=0}^{5} C_{6}^{k} \int \varepsilon^{k} f^{6-k}-\frac{1}{6} \int \varepsilon^{6}
\end{aligned}
$$

Now, we have $\int f_{x}^{2} \leq\|f\|_{H^{1}}^{2},\left|\int f_{x} \varepsilon_{x}\right| \leq\|f\|_{H^{1}}\left\|\varepsilon_{x}\right\|_{L^{2}}, \int f^{6} \leq\|f\|_{H^{1}}^{6}$, and $\left|\int \varepsilon f^{5}\right| \leq\|f\|_{H^{1}}^{5}\|\varepsilon\|_{L^{2}}$. For $k=2, \ldots, 5$, we have the Gagliardo-Nirenberg inequality (whose sharp constant is $\kappa(k)$ ) :

$$
\int \varepsilon^{k} f^{6-k} \leq \kappa(k)\|f\|_{L^{\infty}}^{6-k}\|\varepsilon\|_{L^{2}}^{k / 2+1}\left\|\varepsilon_{x}\right\|_{L^{2}}^{k / 2-1}
$$

For $k=6$, the Gagliardo-Nirenberg inequality also applies, but gives an exponent 2 for $\|\varepsilon\|_{L^{2}}$ :

$$
\frac{1}{6} \int \varepsilon^{6} \leq \frac{\kappa(k)}{6}\|\varepsilon\|_{L^{2}}^{4}\left\|\varepsilon_{x}\right\|_{L^{2}}^{2} \leq \frac{1}{6} \int \varepsilon_{x}^{2} .
$$


So that we get from the energy equality :

$$
\begin{aligned}
\frac{1}{2} \int \varepsilon_{x}^{2} \leq E(f+\varepsilon)+\|f\|_{H^{1}}^{2}+ & \|f\|_{H^{1}}\left\|\varepsilon_{x}\right\|_{L^{2}}+\|f\|_{H^{1}}^{6}+\|f\|_{H^{1}}^{5}\|\varepsilon\|_{L^{2}} \\
& +\sum_{k=2}^{5}\|f\|_{L^{\infty}}^{6-k}\|\varepsilon\|_{L^{2}}^{k / 2+1}\left\|\varepsilon_{x}\right\|_{L^{2}}^{k / 2-1}+\frac{1}{6} \int \varepsilon_{x}^{2} .
\end{aligned}
$$

This can be rewritten as $\left(\|\varepsilon\|_{L^{2}} \leq 1\right)$

$$
\frac{1}{3}\left\|\varepsilon_{x}\right\|_{L^{2}}^{2} \leq E(f+\varepsilon)+2^{5}\left(\|f\|_{H^{1}}+\|f\|_{L^{\infty}}^{6}\right)\left(1+\left\|\varepsilon_{x}\right\|_{L^{2}}^{3 / 2}\right) .
$$

If $a^{2} \leq K_{1}+K_{2} a^{3 / 2}$, then obviously $a^{2} \leq K_{1}+K_{2}^{4}$, so that we get

$$
\left\|\varepsilon_{x}\right\|_{L^{2}}^{2} \leq 3 E(f+\varepsilon)+3 \cdot 2^{6}\left(\|f\|_{H^{1}}+\|f\|_{L^{\infty}}^{24}\right) .
$$

\subsection{Estimates of $U(t) V$ on the right}

We now obtain bounds for $U(t) V$ on the right, which is will be crucial for the monotonicity Lemma 5 , and also in Section 5 (analysis of the interaction of the linear term $U(t) V)$.

Lemma $2\left(U(t) V\right.$ estimates on the right). Let $f \in L^{2}$, then

$$
\|U(t) f\|_{L^{2}\left(1-\psi_{-1}(t)\right)} \leq\|f\|_{L^{2}\left(1-\psi_{-1}(t / 2)\right)} \rightarrow 0 \quad \text { as } \quad t \rightarrow \infty .
$$

In particular, if $f \in H^{1}$, then

$$
\sup _{x \geq m_{-1}(t)}|U(t) f(x)|^{2} \leq 4\|f\|_{L^{2}}\left\|f_{x}\right\|_{L^{2}\left(1-\psi_{-1}(t / 2)\right)} \rightarrow 0 \quad \text { as } \quad t \rightarrow \infty .
$$

Suppose that $\left(1+x_{+}^{q}\right) f(x) \in L^{2}(d x)$, for some $q>0$. Then there exists a constant $C=C\left(\sigma_{0}, x_{1}\right)$ independent of $f$ such that

$$
\forall t \geq 1, \quad\|U(t) f\|_{L^{2}\left(1-\psi_{0}(t)\right)} \leq \frac{C}{t^{q}}\left\|\left(1+x_{+}^{q}\right) f(x)\right\|_{L^{2}(d x)} .
$$

If $\left(1+x_{+}^{1 / 2}\right) f(x) \in L^{2}(d x)$, we have furthermore that

$$
\int_{t \geq 0}\|U(t) f\|_{L^{2}\left(1-\psi_{0}(t)\right)}^{2} d t \leq C\left\|\left(1+x_{+}^{1 / 2}\right) f(x)\right\|_{L^{2}(d x)}^{2}<\infty .
$$

We will apply this result to $V$ and $V_{x}$.

Proof. The key remark is that $U(t)$ "pushes" the $L^{2}$-mass on the left. Let $\varphi=$ $\psi_{-1}$ or $\varphi=\psi_{0}$. We compute :

$$
\begin{aligned}
& \frac{d}{d \tau} \int|U(2 \tau-t) f|^{2} \varphi(\tau) \\
& =2 \int(U(2 \tau-t) f)_{\tau} U(2 \tau-t) f \varphi(\tau)+\int|U(2 \tau-t) f|^{2} \varphi_{\tau}(\tau) \\
& =-4 \int U(2 \tau-t) f_{x x x} U(2 \tau-t) f \varphi(\tau)+\int|U(2 \tau-t) f|^{2} \varphi_{\tau}(\tau)
\end{aligned}
$$




$$
\begin{aligned}
=4 \int U(2 \tau-t) f_{x x} U(2 \tau-t) f_{x} \varphi(\tau)+4 \int U(2 \tau-t) f_{x x} U(2 \tau-t) f \varphi_{x}(\tau) \\
\quad+\int|U(2 \tau-t) f|^{2} \varphi_{\tau}(\tau) \\
=-6 \int\left|U(2 \tau-t) f_{x}\right|^{2} \varphi_{x}(\tau)-4 \int U(2 \tau-t) f_{x} U(2 \tau-t) f \varphi_{x x}(\tau) \\
\quad+\int|U(2 \tau-t) f|^{2} \varphi_{\tau}(\tau) \\
\quad-6 \int\left|U(2 \tau-t) f_{x}\right|^{2} \varphi_{x}(\tau)+\int|U(2 \tau-t) f|^{2}\left(2 \varphi_{x x x}(\tau)+\varphi_{\tau}(\tau)\right) .
\end{aligned}
$$

As $\psi_{x x x} \leq \frac{\sigma_{0}}{4}\left|\psi_{x}\right|$, and $\psi_{x}<0$, we have that, for $\varphi=\psi_{-1}$ or $\psi_{0}$,

$$
\varphi_{x}(\tau)<0 \quad \text { and } \quad 2 \varphi_{x x x}(\tau)+\varphi_{\tau}(\tau) \geq 0 .
$$

So that $\tau \mapsto \int U(2 \tau-t) f(x)^{2} \varphi_{0}(\tau, x) d x$ is an increasing function of $\tau$. In particular, when comparing for $\tau=t$ and $\tau=t / 2(t \geq 0)$, we have :

$$
\forall t \geq 0, \quad \int|U(t) f|^{2} \varphi(t) \geq \int f^{2} \varphi_{0}(t / 2) .
$$

As the flow $U(t)$ preserves the $L^{2}$-mass, we get in each case $\varphi=\psi_{-1}$ or $\psi_{0}$ :

$$
\begin{array}{r}
\forall t \geq 0, \quad \int|U(t) f|^{2}(x)\left(1-\psi_{-1}(t, x)\right) d x \leq \int f^{2}(x)\left(1-\psi_{-1}(t / 2, x)\right) d x, \\
\int|U(t) f|^{2}(x)\left(1-\psi_{0}(t, x)\right) d x \leq \int f^{2}(x)\left(1-\psi_{0}(t / 2, x)\right) d x .
\end{array}
$$

(25) immediatly gives (21). Let $x \geq m_{-1}(t)$. Then for $y \geq x, 1-\psi_{-1}(t, y) \geq$ $1-\frac{2}{\pi} \arctan (1)=\frac{1}{2}$. Thus,

$$
\begin{aligned}
|U(t) f(x)|^{2} & =-2 \int_{y}^{\infty} U(t) f(y) U(t) f_{x}(y) d y \\
& \leq 2\left(\int_{y}^{\infty}|U(t) f(y)|^{2} d y\right)^{1 / 2}\left(\int_{y}^{\infty}\left|U(t) f_{x}(y)\right|^{2} d y\right)^{1 / 2} \\
& \leq 8\left(\int_{y}^{\infty}|U(t) f(y)|^{2}\left(1-\psi_{-1}(t, y)\right) d y\right)^{1 / 2} \\
& \times\left(\int_{y}^{\infty}\left|U(t) f_{x}(y)\right|^{2}\left(1-\psi_{-1}(t, y)\right) d y\right)^{1 / 2} \\
& \leq 8\|U(t) f\|_{L^{2}\left(1-\psi_{-1}(t)\right)}\left\|U(t) f_{x}\right\|_{L^{2}\left(1-\psi_{-1}(t)\right)} \\
& \leq 8\|f\|_{L^{2}\left(1-\psi_{-1}(t / 2)\right)}\left\|f_{x}\right\|_{L^{2}\left(1-\psi_{-1}(t / 2)\right)}
\end{aligned}
$$

This is (22).

We will now use (26). Suppose that for some $q>0,\left(1+x_{+}^{q}\right) f(x) \in L^{2}(d x)$. Then for $t \geq 1$,

$$
\int f^{2}\left(1-\psi_{0}(t / 2)\right)=\int_{x \leq \sigma_{0} t / 4} f^{2}\left(1-\psi_{0}(t / 2)\right)+\int_{x \geq \sigma_{0} t / 4} f^{2}\left(1-\psi_{0}(t / 2)\right)
$$




$$
\begin{aligned}
& \leq \sup _{x \leq \sigma_{0} t / 4}\left(1-\psi_{0}(t / 2, x)\right) \int f^{2}+\left(\frac{\sigma_{0} t}{4}\right)^{-2 q} \int_{x \geq \sigma_{0} t / 4} x^{2 q} f^{2} \\
& \leq C\left(x_{0}\right) e^{-\frac{\sigma_{0} \sqrt{\sigma_{0}}}{4}} t\|f\|_{L^{2}}^{2}+C\left(\sigma_{0}\right) t^{-2 q}\left\|x_{+}^{q} f\right\|_{L^{2}}^{2} .
\end{aligned}
$$

And we get

$$
\forall t \geq 1, \quad\|U(t) f\|_{L^{2}\left(1-\psi_{0}(t)\right)} \leq \frac{C}{t^{q}}\left\|\left(1+x_{+}\right)^{q} f\right\|_{L^{2}},
$$

which is $(23)$.

Suppose now that $\left(1+x_{+}^{1 / 2}\right) f(x) \in L^{2}(d x)$. Then

$$
\begin{aligned}
\int_{t=0}^{\infty} \int_{x}|U(t) f(x)|^{2}\left(1-\psi_{0}(t, x)\right) d x d t & \leq \int_{t=0}^{\infty} \int_{x} f^{2}(x)\left(1-\psi_{0}(t / 2, x)\right) d x d t \\
& \leq \int_{x} f^{2}(x) \int_{t=0}^{\infty}\left(1-\psi_{0}(t / 2, x)\right) d t d x \\
& \leq C \int f^{2}(x) \frac{1+x_{+}}{\sigma_{0}} d x \\
& \leq C\left\|\left(1+x_{+}^{1 / 2}\right) f(x)\right\|_{L^{2}(d x)}^{2},
\end{aligned}
$$

and this proves $(24)$.

\section{Control of the interaction of $w_{n}(t)$ with the soli- tons}

This section is devoted to the proof of Proposition 2. We develop arguments very similar to those of [17] and [16].

\subsection{Modulation close to the asymptotic profile}

Lemma 3. There exist $T_{1}$ large enough and $\varepsilon_{1}>0$ small enough such that if $T_{1} \geq T_{1}$ and $\varepsilon_{0} \leq \varepsilon_{1}$, the following is true.

There exist $2 N C^{1}$ functions $y_{j}, \gamma_{j}:\left[I_{n}, S_{n}\right] \rightarrow \mathbb{R}$ such that if we denote:

$$
\begin{aligned}
& \tilde{R}_{j}(t, x)=Q_{\gamma_{j}(t)}\left(x-y_{j}(t)\right), \quad \tilde{R}(t, x)=\sum_{j=1}^{N} \tilde{R}_{j}(t, x), \\
\tilde{w}_{n}(t)= & u_{n}(t, x)-U(t) V-\tilde{R}(t, x),
\end{aligned}
$$

we have for all $j=1, \ldots, N$ :

$$
\int \tilde{w}_{n}(t, x) \tilde{R}_{j_{x}}(t, x) d x=0 \quad \text { and } \quad \int \tilde{w}_{n}(t, x) \tilde{R}_{j}{ }^{3}(t, x) d x=0 .
$$

Moreover, there exists $C_{11}$ such that:

$$
\left\|\tilde{w}_{n}(t)\right\|_{L^{2}}+\sum_{j=1}^{N}\left|\gamma_{j}(t)-c_{j}\right|+\sum_{j=1}^{N}\left|y_{j}(t)-x_{j}-c_{j} t\right| \leq C_{11} \varepsilon_{0},
$$




$$
\begin{aligned}
\left|y_{j}^{\prime}(t)-c_{j}\right|+\left|\gamma_{j}^{\prime}(t)\right| \leq C_{11} e^{-\frac{\sigma_{0} \sqrt{\sigma_{0}}}{2} t} & +C_{11}\|U(t) V\|_{L^{2}\left(1-\psi_{0}(t)\right)} \\
& +C_{11}\left(\int{\tilde{w_{n}}}^{2}(t) e^{-\sqrt{\sigma_{0}}\left|x-c_{j} t\right|}\right)^{1 / 2} .
\end{aligned}
$$

Proof. The existence of the modulation is essentially an application of the implicit function theorem. Consider the $C^{\infty}$ functional

$$
\begin{aligned}
F: & {\left[I_{n}, S_{n}\right] \times L^{2} \times \mathbb{R}^{n} \times \mathbb{R}^{n} \rightarrow \mathbb{R}^{n} \times \mathbb{R}^{n}, } \\
& \left(t, u,\left(y_{j}\right)_{j},\left(\gamma_{j}\right)_{j}\right) \mapsto\left(F_{1 j}\left(t, u,\left(y_{j}\right)_{j},\left(\gamma_{j}\right)_{j}\right), F_{2 j}\left(t, u,\left(y_{j}\right)_{j},\left(\gamma_{j}\right)_{j}\right)\right),
\end{aligned}
$$

with

$$
\begin{aligned}
& F_{1 j}\left(t, u,\left(y_{j}\right)_{j},\left(\gamma_{j}\right)_{j}\right)=\int(u-U(t) V-\tilde{R}(t)) \tilde{R}_{j_{x}}(t) d x, \\
& F_{2 j}\left(t, u,\left(y_{j}\right)_{j},\left(\gamma_{j}\right)_{j}\right)=\int(u-U(t) V-\tilde{R}(t)) \tilde{R}_{j}^{3}(t) d x,
\end{aligned}
$$

locally on a neighborhood of the curve $y_{j}(t)=x_{j}+c_{j} t, \gamma_{j}(t)=c_{j}, u=$ $U(t) V+R(t)$. To express $y_{j}, \gamma_{j}$ in function of $u, t$, we apply the implicit function theorem stated in the Appendix : let us prove that $\partial_{y_{j}, \gamma_{j}} F$ is invertible at points $\left.\left(t, U(t) V+R(t), x_{j}+c_{j} t\right)_{j},\left(c_{j}\right)_{j}\right)$, compute $\partial_{u} F$, and do some uniform (in $t$ ) estimates.

For all $t, \alpha$ being $y_{j}$ or $\gamma_{k}$ we compute

$$
\begin{aligned}
& \partial_{\alpha} F_{1 j}(t)=-\int\left(\partial_{\alpha} \tilde{R}_{j}\right)(t) \tilde{R}_{j_{x}}(t)+\int\left(u-U(t) V-\tilde{R}_{j}(t)\right)\left(\partial_{\alpha} \tilde{R}_{j_{x}}\right)(t), \\
& \partial_{\alpha} F_{2 j}(t)=\int\left(\partial_{\alpha} \tilde{R}_{j}\right)(t) \tilde{R}_{j}^{3}(t)+3 \int\left(u-U(t) V-\tilde{R}_{j}(t)\right)\left(\partial_{\alpha} \tilde{R}_{j}\right)(t) \tilde{R}_{j}^{2}(t),
\end{aligned}
$$

and

$$
\begin{aligned}
& \left(\partial_{y_{j}} \tilde{R}_{j}\right)(t, x)=-\tilde{R}_{j_{x}}(t, x), \\
& \left(\partial_{\gamma_{j}} \tilde{R}_{j}\right)(t, x)=\frac{1}{4 c_{j}} \tilde{R}_{j}(t, x)+\frac{1}{2 c_{j}}\left(x-x_{j}-c_{j} t\right) \tilde{R}_{j_{x}}(t, x) .
\end{aligned}
$$

Let $u, y_{j}$ and $\gamma_{j}$ be such that

$$
\|u-U(t) V-R(t)\|_{L^{2}}+\sum_{j=1}^{N}\left|y_{j}\right|+\left|\gamma_{j}\right| \leq \varepsilon_{0} .
$$

We get that

$$
\left|\partial_{y_{j}} F_{1 j}-\int Q_{c_{j} x}^{2}\right| \leq C \varepsilon_{0}
$$

(recall $\left\|Q_{c_{j}}\right\|_{L^{2}}=\|Q\|_{L^{2}}$ ) and for $k \neq j$, using the exponential decay :

$$
\begin{aligned}
\left|\partial_{y_{k}} F_{1 j}(t)\right| & \leq C e^{-\frac{\sigma \sqrt{\sigma_{0}}}{4} t}+C \varepsilon_{0}, \\
\left|\partial_{y_{k}} F_{2 j}(t)\right|+\left|\partial_{\gamma_{k}} F_{1 j}(t)\right|+\left|\partial_{\gamma_{k}} F_{2 j}(t)\right| & \leq C e^{-\frac{\sigma \sqrt{\sigma_{0}}}{4} t}+C \varepsilon_{0} .
\end{aligned}
$$

Now $Q$ is an even function, so that

$$
\left|\partial_{\gamma_{j}} F_{1 j}(t)\right| \leq C \delta, \quad\left|\partial_{y_{j}} F_{2 j}(t)\right| \leq C \varepsilon_{0} .
$$


Finally, for $\partial_{\gamma_{j}} F_{2 j}$, we have

$$
\left|\partial_{\gamma_{j}} F_{2 j}-\frac{1}{4 c_{j}} \int Q_{c_{j}}{ }^{4}\right| \leq C \varepsilon_{0} .
$$

Hence, for $T_{1}$ large enough and $\varepsilon_{1}>0$ small enough, the conditions of the implicit function theorem are fufilled, and we obtain the existence and regularity of $y_{j}(t), \gamma_{j}(t)$, along with the first estimate $(27)$.

For the second estimate (28), we compute the equation satisfied by $\tilde{w}(t)$ and do the scalar product with every $\tilde{R}_{j_{x}}$ and every $\tilde{R}_{j}^{3}$ : the relations obtained in this way will yield the result. The equation satisfied by $\tilde{R}_{k}$ (using $-c_{k} R_{k x}+$ $\left.R_{k x x x}+R_{k}^{5}\right)_{x}=0$ ) is now

$$
\begin{aligned}
& \tilde{R}_{k t}+\tilde{R}_{k x x x} \\
& =\left(-y_{k}^{\prime}(t)+c_{k}\right) \tilde{R}_{k x}+\frac{\gamma_{k}^{\prime}(t)}{\gamma_{k}(t)}\left(\frac{\tilde{R}_{k}(t)}{4}+\left(x-y_{k}(t)\right) \frac{\tilde{R}_{k x}(t)}{2}\right)-c_{k} \tilde{R}_{k x}+\tilde{R}_{k x x x} \\
& =\left(-y_{k}^{\prime}(t)+c_{k}\right) \tilde{R}_{k x}+\frac{\gamma_{k}^{\prime}(t)}{\gamma_{k}(t)}\left(\frac{\tilde{R}_{k}(t)}{4}+\left(x-y_{k}(t)\right) \frac{\tilde{R}_{k x}(t)}{2}\right)-\left(\tilde{R}_{k}^{5}\right)_{x} .
\end{aligned}
$$

So that with $\tilde{w}_{n}=u_{n}(t)-U(t) V-\tilde{R}(t)$, we get

$$
\begin{array}{r}
\tilde{w}_{n t}+\tilde{w}_{n x x x}=\sum_{k=1}^{N}\left(y_{k}^{\prime}(t)-c_{k}\right) \tilde{R}_{k x} \\
-\sum_{k=1}^{N} \frac{\gamma_{k}^{\prime}}{\gamma_{k}}\left(\frac{\tilde{R}_{k}}{4}+\left(x-y_{k}(t)\right) \frac{\tilde{R}_{k x}}{2}\right) \\
-\left(\left(\tilde{w}_{n}+U(t) V+\tilde{R}\right)^{5}-\sum_{k=1}^{N} \tilde{R}_{k}^{5}\right)_{x} .
\end{array}
$$

Now, if we express $\tilde{R}_{j}$ in terms of $R_{j}$, we get

$$
\tilde{R}_{j_{x t}}=-y_{j}^{\prime}(t) \tilde{R}_{j_{x x}}+\frac{\gamma_{j}^{\prime}(t)}{\gamma_{j}(t)}\left(\frac{\tilde{R}_{j_{x}}(t)}{4}+\left(x-y_{j}(t)\right) \frac{\tilde{R}_{j_{x x}}(t)}{2}+\frac{\tilde{R}_{j_{x}}(t)}{2}\right) .
$$

And keeping in mind that $\frac{d}{d t} \int \tilde{w}_{n} \tilde{R}_{j_{x}}=\int \tilde{w}_{n} \tilde{R}_{j_{x}}=0$, we get

$$
\int \tilde{w}_{n t} \tilde{R}_{j_{x}}=-\int \tilde{w}_{n} \tilde{R}_{j x t}=\int \tilde{w}_{n}\left(y_{j}^{\prime}(t)-\frac{\gamma_{j}^{\prime}(t)}{\gamma_{j}(t)} \frac{x-y_{j}(t)}{2}\right) \tilde{R}_{j_{x x}} .
$$

We multiply (29) by $\tilde{R}_{j_{x}}$, integrate in $x$, and do integration by parts :

$$
\begin{aligned}
& \left(y_{j}^{\prime}(t)-c_{j}\right) \int \tilde{R}_{j_{x}}^{2} \\
& =-y_{j}^{\prime}(t) \int \tilde{w}(t) \tilde{R}_{j_{x x}}+\frac{\gamma_{j}^{\prime}(t)}{2 \gamma_{j}(t)} \int \tilde{w}_{n}(t)\left(x-y_{k}(t)\right) \tilde{R}_{j_{x x}}-\int \tilde{w}_{n}(t) \tilde{R}_{j_{x x x x}} \\
& -\sum_{k, k \neq j}\left(c_{k}-y_{k}^{\prime}(t)\right) \int \tilde{R}_{j_{x}} \tilde{R}_{k x}+\sum_{k=1}^{N} \frac{\gamma_{k}^{\prime}}{\gamma_{k}} \int \tilde{R}_{j_{x}}\left(\frac{\tilde{R}_{k}}{4}+\left(x-y_{k}(t)\right) \frac{\tilde{R}_{k x}}{2}\right) \\
& \quad-\int\left(\left(\tilde{w}_{n}+U(t) V+\tilde{R}\right)^{5}-\sum_{k=1}^{N} \tilde{R}_{k}^{5}\right) \tilde{R}_{j_{x x} .}
\end{aligned}
$$


First consider the three first terms : as $Q_{x x}=Q-Q^{p}$, we can express $\tilde{R}_{j_{x x}}$ and $\tilde{R}_{j_{x x x x}}$ in terms of powers of $\tilde{R}_{j}$. Therefore, the integral part of these terms is bounded by

$$
\int\left|\tilde{w}_{n}(t)\right|\left(1+\left|x-c_{j} t\right|\right) e^{-\sqrt{\sigma_{0}}\left|x-c_{j} t\right|} \leq C\left(\int\left|\tilde{w}_{n}(t)\right|^{2} e^{-\sqrt{\sigma_{0}}\left|x-c_{j} t\right|}\right)^{1 / 2} .
$$

For the fourth term, $\int\left|R_{j_{x}} R_{k x}\right| \leq e^{-\frac{\sigma_{0} \sqrt{\sigma_{0}}}{2}} t$. This also apply to the fifth term, but for $j$-term, which vanishes:

$$
\int \tilde{R}_{j_{x}}\left(\frac{\tilde{R}_{j}}{4}+\left(x-y_{j}(t)\right) \frac{\tilde{R}_{j_{x}}}{2}\right)=0 .
$$

And for the non-linear last term, when developing, the large terms cancel one another, so that we can control the rest by :

$$
C \int\left(\left|\tilde{w}_{n}(t)\right|+|U(t) V|\right) e^{-\sqrt{\sigma_{0}}\left|x-c_{j} t\right|} .
$$

Finally, we have altogether

$$
\begin{aligned}
\left|y_{j}^{\prime}(t)-c_{j}\right| \leq C & \left(1+\left|\frac{\gamma_{j}^{\prime}(t)}{\gamma_{j}(t)}\right|\right)\left(\int\left|\tilde{w}_{n}(t)\right|^{2} e^{-\sqrt{\sigma_{0}}\left|x-c_{j} t\right|}\right)^{1 / 2} \\
& +C e^{-\frac{\sigma_{0} \sqrt{\sigma 0}}{2} t} \sum_{k, k \neq j}\left|y_{k}^{\prime}(t)-c_{k}\right|+C e^{-\frac{\sigma_{0} \sqrt{\sigma 0}}{2} t} \sum_{k, k \neq j}\left|\frac{\gamma_{k}^{\prime}(t)}{\gamma_{k}(t)}\right| \\
& +C e^{-\frac{\sigma_{0} \sqrt{\sigma 0}}{2} t}+C\|U(t) V\|_{L^{2}\left(1-\psi_{0}(t)\right) .}
\end{aligned}
$$

Now, we have to do the same kind of argument on $\gamma_{j}$. As

$$
\tilde{R}_{j_{t}}=-y_{j}^{\prime}(t) \tilde{R}_{j_{x}}+\frac{\gamma_{j}^{\prime}(t)}{\gamma_{j}(t)}\left(\frac{\tilde{R}_{j}(t)}{4}+\left(x-y_{j}(t)\right) \frac{\tilde{R}_{j_{x}}(t)}{2}+\frac{\tilde{R}_{j}(t)}{2}\right),
$$

we have

$$
\int \tilde{w}_{n t} \tilde{R}_{j}^{3}=-3 \int \tilde{w}_{n} \tilde{R}_{j_{t}} \tilde{R}_{j}^{2}=3 \int\left(\left(y_{j}^{\prime}-\frac{\gamma_{j}^{\prime}}{2 \gamma_{j}}\left(x-y_{j}\right)\right)\left(\tilde{w}_{n} \tilde{R}_{j_{x}} \tilde{R}_{j}^{2}\right) .\right.
$$

Let us multiply (29) by $\tilde{R}_{j}^{3}$. We obtain, after an integration by parts $\int(x-$ $\left.y_{j}(t)\right) \tilde{R}_{j} \tilde{R}_{j_{x}}=-\frac{1}{2} \int \tilde{R}_{j}^{2}$,

$$
\begin{gathered}
\frac{1}{4} \frac{\gamma_{j}^{\prime}(t)}{\gamma_{j}(t)} \int \tilde{R}_{j}^{4}=\frac{\gamma_{j}^{\prime}(t)}{2 \gamma_{j}(t)} \int \tilde{w}_{n}(t)\left(x-y_{k}(t)\right) \tilde{R}_{j}{ }^{3}-\int \tilde{w}_{n}(t)\left(\tilde{R}_{j}{ }^{3}\right)_{x x x} \\
-\sum_{k=1}^{N}\left(c_{k}-y_{k}^{\prime}\right) \int \tilde{R}_{j}{ }^{3} \tilde{R}_{k x}+\sum_{k \neq j} \frac{\gamma_{k}^{\prime}}{\gamma_{k}} \int \tilde{R}_{j}{ }^{3}\left(\frac{\tilde{R}_{k}}{4}+\left(x-y_{k}(t)\right) \frac{\tilde{R}_{k x}}{2}\right) \\
-3 \int\left(\left(\tilde{w}_{n}+U(t) V+\tilde{R}\right)^{5}-\sum_{k, k \neq j} \tilde{R}_{k}^{5}\right) \tilde{R}_{j} \tilde{R}_{j}{ }^{2} .
\end{gathered}
$$


Let us notice again that the only possibly large term (in the first sum) is in fact $0\left(\int \tilde{R}_{j}^{3} \tilde{R}_{j_{x}}=0\right)$. If we argue like before, we get

$$
\begin{aligned}
\left|\frac{\gamma_{j}^{\prime}(t)}{\gamma_{j}(t)}\right| & \leq C\left(1+\frac{\gamma_{j}^{\prime}(t) \mid}{\gamma_{j}(t)}\right)\left(\int \tilde{w}_{n}^{2}(t) e^{-\sqrt{\sigma_{0}}\left|x-c_{j} t\right|}\right)^{1 / 2} \\
& +C e^{-\frac{\sigma_{0} \sqrt{\sigma_{0}}}{2} t} \sum_{k, k \neq j}\left|y_{k}^{\prime}(t)-c_{k}\right|+e^{-\frac{\sigma_{0} \sqrt{\sigma_{0}}}{2}} t \sum_{k, k \neq j}\left|\frac{\gamma_{k}^{\prime}(t)}{\gamma_{k}(t)}\right| \\
& +C e^{-\frac{\sigma_{0} \sqrt{\sigma_{0}}}{2} t}+C\|U(t) V\|_{L^{2}\left(1-\psi_{0}(t)\right) .}
\end{aligned}
$$

We can now use our computations. Let us sum our $2 N$ estimates (30) and (31) together :

$$
\begin{aligned}
& \sum_{k=1}^{N}\left(\left|y_{k}^{\prime}(t)-c_{k}\right|+\left|\frac{\gamma_{k}^{\prime}(t)}{\gamma_{k}(t)}\right|\right) \leq C\left(1+\sum_{k=1}^{N}\left|y_{k}^{\prime}(t)\right|+\sum_{k=1}^{N}\left|\frac{\gamma_{k}^{\prime}(t)}{\gamma_{k}(t)}\right|\right)\left\|\tilde{w}_{n}\right\|_{L^{2}} \\
& +C e^{-\frac{\sigma_{0} \sqrt{\sigma 0}}{2}} t\left(\sum_{k=1}^{N}\left|y_{k}^{\prime}(t)\right|+\left|\frac{\gamma_{k}^{\prime}(t)}{\gamma_{k}(t)}\right|\right)+C e^{-\frac{\sigma_{0} \sqrt{\sigma 0}}{2} t}+C\|U(t) V\|_{L^{2}\left(1-\psi_{0}(t)\right)} .
\end{aligned}
$$

So that for $\varepsilon_{1}$ small enough, as $\left\|\tilde{w}_{n}\right\|_{L^{2}} \leq \varepsilon_{0} \leq \varepsilon_{1}$, and $t \geq T_{1}$ large enough, we get

$$
\sum_{k=1}^{N}\left|y_{k}^{\prime}(t)-c_{k}\right|+\left|\frac{\gamma_{k}^{\prime}(t)}{\gamma_{k}(t)}\right| \leq C .
$$

Let us now go back to (30) : we get exactly what we want on $\left|y_{j}^{\prime}(t)-c_{j}\right|$. In the same way, as $\gamma_{k}>\sigma_{0}$ for $\varepsilon_{0}$ small enough (first estimate), we get the result for $\left|\gamma_{j}^{\prime}(t)\right|$ by plugging in (31).

Notice that $\left\|w_{n}(t)-\tilde{w}_{n}(t)\right\|_{H^{s}}=\|R(t)-\tilde{R}(t)\|_{H^{s}} \leq C(s)\left(\left|y_{j}(t)-c_{j} t-x_{j}\right|+\left|\gamma_{j}(t)-c_{j} t\right|\right)$.

We now turn to the extraction of the main terms in $\int u_{n}{ }^{2}(t)$ and $E\left(u_{n}(t)\right)$, which writes as follows : remind $\left\|Q_{c}\right\|_{L^{2}}=\|Q\|_{L^{2}}$ and $\left.E\left(Q_{c}\right)=0\right)$. Let us denote for simplicity :

$$
\tilde{v_{n}}(t)=\tilde{w_{n}}(t)+U(t) V=u_{n}(t)-\tilde{R}(t) .
$$

Lemma 4 (Main terms in $M_{j}$ and $E_{j}, j \geq 1$ ). We have, for all $t \in\left[I_{n}, S_{n}\right]$,

$$
\begin{aligned}
& \left|M_{j}(t)-\left(\int Q^{2}+2 \int \tilde{v_{n}}(t) \tilde{R}_{j}(t)+\int{\tilde{v_{n}}}^{2}(t) \phi_{j}(t)\right)\right| \leq C_{12} e^{-\frac{\sigma_{0} \sqrt{\sigma_{0}}}{2} t}, \\
& \text { (2) }\left|E_{j}(t)-\left[\frac{1}{2} \int\left({\tilde{v_{n}}}^{2}(t)-5 \tilde{R}_{j}^{4}(t){\tilde{v_{n}}}^{2}(t)\right) \phi_{j}(t)-\gamma_{j}(t) \int \tilde{v_{n}}(t) \tilde{R}_{j}(t)\right)\right| \\
& \leq C_{12} e^{-\frac{\sigma_{0} \sqrt{\sigma_{0}}}{2} t}+C_{12} \varepsilon_{0} \int{\tilde{v_{n}}}^{2}(t) \phi_{j}(t), \\
& \text { (3) }\left|\left(E_{j}(t)+\frac{\gamma_{j}(t)}{2} M_{j}(t)\right)-\frac{\gamma_{j}(t)}{2} \int Q^{2}-\frac{1}{2} H_{j}(t)\right| \\
& \leq C_{12} e^{-\frac{\sigma_{0} \sqrt{\sigma_{0}}}{2} t}+C_{12} \varepsilon_{0} \int{\tilde{v_{n}}}^{2}(t) \phi_{j}(t),
\end{aligned}
$$

where $H_{j}(t)=\int\left({\tilde{v_{n}}}^{2}(t)-5 \tilde{R}_{j}^{4}(t){\tilde{v_{n}}}^{2}(t)+\gamma_{j}(t){\tilde{v_{n}}}^{2}(t)\right) \phi_{j}(t)$. 
Proof. (1) We compute $\left(u_{n}=\tilde{v_{n}}+\tilde{R}\right)$ :

$$
M_{j}(t)=\int u_{n}{ }^{2} \phi_{j}(t)=\int\left({\tilde{v_{n}}}^{2}+2 \tilde{v_{n}} \tilde{R}(t)+\sum_{k=1}^{N}{\tilde{R_{k}}}^{2}(t)\right) \phi_{j}(t) .
$$

As $\phi_{j}(t)$ localized in the interval $\left[m_{j-1}(t), m_{j}(t)\right]$, like $\tilde{R}_{j}(t)$ we get $(k \neq j)$

$$
\left|\int \tilde{R}_{j}^{2}(t) \phi_{j}(t)-\int Q_{\gamma_{j}(t)}^{2}\right| \leq C e^{-\frac{\sigma_{0} \sqrt{\sigma_{0}}}{2} t}, \quad \int \tilde{R}_{k}^{2}(t) \phi_{j}(t) \leq C e^{-\frac{\sigma_{0} \sqrt{\sigma_{0}}}{2} t} .
$$

(2) In the same way,

$$
\begin{aligned}
E_{j}(t)= & \int\left(\frac{1}{2}\left({\tilde{v_{n}}}^{2}(t)+2 \tilde{v_{n x}}(t) \tilde{R}_{x}+\tilde{R}_{x}^{2}\right)-\frac{1}{6}\left(\tilde{v_{n}}(t)+\tilde{R}(t)\right)^{6}\right) \phi_{j}(t) \\
= & \int\left(\frac{1}{2}{\tilde{v_{n}}}^{2}(t)-\frac{5}{2} \tilde{R}^{4}{\tilde{v_{n}}}^{2}(t)\right) \phi_{j}+\int\left(\frac{1}{2} \tilde{R}_{x}^{2}-\frac{1}{6} \tilde{R}^{6}\right) \phi_{j}(t) \\
& -\int \tilde{v_{n}}(t)\left(\tilde{R}_{x x}+\tilde{R}^{5}\right) \phi_{j}-\int \tilde{R}_{x} \tilde{v_{n}}(t) \phi_{j_{x}} \\
& +\int\left[\frac{\left(-\left(\tilde{v_{n}}(t)+\tilde{R}\right)^{6}+\tilde{R}^{6}\right)}{6}+\tilde{v_{n}}(t) \tilde{R}^{p}+\frac{5}{2} \tilde{R}^{4}{\tilde{v_{n}}}^{2}(t)\right] \phi_{j} .
\end{aligned}
$$

We keep the first integral untouched. The second one is $E\left(Q_{\gamma_{j}(t)}\right)$ up to an exponential correction. For the third one, recall that $Q_{x x}+Q^{5}=Q$, so that again

$$
\int \tilde{v_{n}}(t)\left(\tilde{R}_{x x}+\tilde{R}^{5}\right) \phi_{j}=\gamma_{j}(t) \int \tilde{v_{n}}(t) \tilde{R}_{j}(t)+O\left(e^{-\frac{\sigma_{0} \sqrt{\sigma_{0}}}{2}} t\right) .
$$

The fourth one is exponentially small (with $\tilde{R}$ and $\phi_{j_{x}}$ ). Finally the fifth is of order at least 3 in $v_{n}$, so that we control it by

$$
\int \tilde{v}(t)^{k} \phi_{j}(t) \leq\|\tilde{v}(t)\|_{L^{\infty}}^{k-2} \int \tilde{v}(t)^{2} \phi_{j}(t) .
$$

This gives the desired result.

(3) is the sum of (1) and (2). Notice that the scalar product $\int \tilde{v}(t) \tilde{R}_{j}(t)$ vanishes in $H_{j}$ : the linear combination has been constructed for this.

Proposition 4 (Positivity of a quadratic form). There exists $\sigma_{1}>0$ small enough and $\lambda_{1}>0$ so that the following is true. For $\sigma_{0} \leq \sigma_{1}$, there exists $T_{1}=T_{1}\left(\sigma_{0}\right)$, so that for all $t \geq T_{1}$, for all $j=1, \ldots, N$, and for all $v \in H^{1}$,

$$
\begin{aligned}
& \int\left(v_{x}^{2}-5 \tilde{R}_{j}(t)^{4} v^{2}+\gamma_{j}(t) v^{2}\right) \phi_{j}(t) \\
& \quad \geq \lambda_{1} \int\left(v_{x}^{2}+v^{2}\right) \phi_{j}(t)-\frac{1}{\lambda_{1}}\left(\left(\int v \tilde{R}_{j}^{3}(t)\right)^{2}+\left(\int v \tilde{R}_{j_{x}}(t)\right)^{2}\right) .
\end{aligned}
$$

Proof. A similar result can be found in [17, Lemma 4] and [16, Appendix A]. For the sake of completeness, the complete proof is done in the Appendix.

From now on and throughout the rest of the proof, $\sigma_{0}<\sigma_{1}$ is fixed. 


\subsection{Almost monotonicity properties and Abel transform}

Lemma 5 (Monotocity formula [12]). There exists $T_{1}$ large enough, $\varepsilon_{1}$ small enough, and $C_{13}>0$ such that for all $j=0, \ldots, N$ and $t \in\left[I_{n}, S_{n}\right]$,

$$
\begin{gathered}
\sum_{k=0}^{j}\left(M_{k}\left(S_{n}\right)-M_{k}(t)\right) \geq-C_{13} e^{-\frac{\sigma_{0} \sqrt{\sigma_{0}}}{4} t}, \\
\sum_{k=0}^{j}\left(F_{k}\left(S_{n}\right)-F_{k}(t)\right) \geq-C_{13} e^{-\frac{\sigma_{0} \sqrt{\sigma_{0}}}{4} t} .
\end{gathered}
$$

Proof. This lemma is very similar to the monotonicity Lemma of [17] and [11]. The only difference is the presence of the term $U(t) V$ : this will be taken care of essentially due to pointwise smallness of $U(t) V$ for $x \geq\left(\sigma_{0} / 2\right) t$, that is (22).

Let us now do the computations. First notice

$$
\sum_{k=0}^{j} M_{k}(t)=\int u_{n t}^{2}(t) \psi_{j}(t), \quad \sum_{k=0}^{j} E_{k}(t)=\int\left(\frac{1}{2} u_{n x}^{2}(t)-\frac{1}{6} u_{n}{ }^{6}(t)\right) \psi_{j}(t) .
$$

For $j=N$, the result is the conservation of mass and energy. Otherwise we compute for $f(t, x) \in C^{3}$ :

$$
\begin{aligned}
\frac{d}{d t} \int u_{n}{ }^{2} f-\int u_{n}{ }^{2} f_{t} & =2 \int u_{n t} u_{n} f=-2 \int\left(u_{n x x}+u_{n}{ }^{5}\right)_{x} u_{n} f \\
& =2 \int\left(u_{n x x}+u_{n}{ }^{p}\right)\left(u_{n x} f+u_{n} f_{x}\right) \\
& =\int\left(-3 u_{n x}^{2}+\frac{5}{3} u_{n}^{6}\right) f_{x}-2 \int u_{n x} u_{n} f_{x x} \\
& =\int\left(-3 u_{n x}^{2}+\frac{5}{3} u_{n}^{6}\right) f_{x}+\int u_{n}{ }^{2} f_{x x x}
\end{aligned}
$$

So that we get

$$
\frac{d}{d t} \int u_{n}{ }^{2} \psi_{j}(t)=-\int\left(3 u_{n_{x}}^{2}+m_{j}^{\prime}(t) u_{n}{ }^{2}-\frac{5}{3} u_{n}{ }^{6}\right) \psi_{j_{x}}+\int u_{n}{ }^{2} \psi_{j x x x} .
$$

But $m_{j}^{\prime}(t) \geq \sigma_{0}$ so that by (18), and $\psi_{j_{x}} \leq 0$ :

$$
\frac{d}{d t} \int u_{n}{ }^{2} \psi_{j}(t) \geq-\int\left(3 u_{n_{x}}^{2}+\frac{3}{4} \sigma_{0} u_{n}{ }^{2}-\frac{5}{3} u_{n}{ }^{6}\right)\left|\psi_{j_{x}}\right|(t) .
$$

It remains to bound the third term. We consider two cases. When $x \in I_{j}(t)=$ $\left[m_{j} t-\frac{\sigma_{0}}{2} t, m_{j}(t)+\frac{\sigma_{0}}{2} t\right], \psi_{j_{x}}$ is big but $R(t)$ and $U(t) V$ are small (recall (22)) so that $u_{n}$ too. More precisely, for $x \in I_{j 1}(t), x \geq m_{-1}(t)$, and

$$
\begin{aligned}
\left|\frac{5}{3} u_{n}{ }^{4}(t, x)\right| & \leq C\left(\left\|w_{n}(t)\right\|_{L^{\infty}}^{4}+\|U(t) V\|_{L^{\infty}\left(x \geq m_{-1}(t)\right)}^{p-1}+|R(t, x)|^{4}\right) \\
& \leq C\left(K_{0}^{2} \varepsilon_{0}^{2}+\|V\|_{L^{2}\left(1-\psi_{-1}(t / 2)\right)}\left\|V_{x}\right\|_{L^{2}\left(1-\psi_{-1}(t / 2)\right)}+e^{-\frac{\sigma_{0} \sqrt{\sigma 0}}{2}} t\right) \\
& \leq \frac{\sigma_{0}}{4}
\end{aligned}
$$


if $T_{1}$ is large enough $\left(t \geq T_{1}\right)$, and $\varepsilon_{1}$ is small enough. On this interval, the second term is larger than the third :

$$
\frac{5}{3} \int_{x \in I_{j}(t)} u_{n}{ }^{6}\left|\psi_{j_{x}}\right|(t) \leq \frac{\sigma_{0}}{4} \int u_{n}{ }^{2}\left|\psi_{j_{x}}\right|(t) .
$$

When $x \in \mathbb{R} \backslash I_{j}(t)$, then $x \notin\left[m_{j}(t)-\frac{\sigma_{0}}{2} t, m_{j}(t)+\frac{\sigma_{0}}{2} t\right]$, so that

$$
\left|\psi_{j_{x}}(t, x)\right| \leq C e^{-\frac{\sigma_{0} \sqrt{\sigma_{0}}}{4} t} .
$$

Now by interpolation between $L^{2}$ and $H^{1}$, we have a uniform control $\int\left|u_{n}\right|^{6} \leq$ $C$ :

So that finally

$$
\frac{5}{3} \int_{x \in \mathbb{R} \backslash I_{j}(t)} u_{n}{ }^{6}\left|\psi_{j_{x}}\right|(t) \leq C e^{-\frac{\sigma_{0} \sqrt{\sigma_{0}}}{4} t}
$$

$$
\frac{d}{d t} \int u_{n}{ }^{2} \psi_{j}(t) \geq \int\left(3 u_{n x}^{2}+\frac{\sigma_{0}}{2} u_{n}^{2}\right)\left|\psi_{j_{x}}(t)\right|-C e^{-\frac{\sigma_{0} \sqrt{\sigma_{0}}}{4} t} \geq-C e^{-\frac{\sigma_{0} \sqrt{\sigma_{0}}}{4} t} .
$$

We integrate this last estimate between $t$ and $S_{n}$, and this gives the estimates on $M_{j}$.

For the estimates on $F_{j}$, we compute in a similar way

$$
\begin{aligned}
\frac{d}{d t} & \int\left(u_{n x}^{2}-\frac{1}{3} u_{n}{ }^{6}\right) f-\int\left(u_{n x}^{2}-\frac{1}{3} u_{n}{ }^{6}\right) f_{t} \\
& =2 \int\left(u_{n x t} u_{n x}-u_{n}{ }^{5} u_{n t}\right) f=-2 \int u_{n t}\left(u_{n x x}+u_{n}{ }^{p}\right) f-2 \int u_{n t} u_{n x} f_{x} \\
& =-\int\left(u_{n x x}+u_{n}{ }^{5}\right)^{2} f_{x}+2 \int\left(u_{n x x}+u_{n}{ }^{5}\right)_{x} u_{n x} f_{x} \\
& =-\int\left(\left(u_{n x x}+u_{n}{ }^{5}\right)^{2}+2 u_{n x x}^{2}-10 u_{n x}^{2} u_{n}{ }^{p-1}\right) f_{x}-2 \int u_{n x x} u_{n x} f_{x x} \\
& =-\int\left(\left(u_{n x x}+u_{n}{ }^{p}\right)^{2}+2{u_{n x x}}_{x}^{2}-10 u_{n_{x}}^{2} u_{n}{ }^{p-1}\right) f_{x}+\int u_{n x}^{2} f_{x x x} .
\end{aligned}
$$

So that

$$
\begin{aligned}
& \frac{d}{d t} \int\left(u_{n_{x}}^{2}-\frac{1}{3} u_{n}{ }^{6}\right) \psi_{j}(t) \\
& =-\int\left(\left(u_{n x x}+u_{n}^{5}\right)^{2}+2 u_{n x x}^{2}-10 u_{n_{x}}^{2} u_{n}{ }^{4}\right) \psi_{j_{x}}(t) \\
& \quad-m_{j}^{\prime}(t) \int\left(u_{n_{x}}^{2}-\frac{1}{3} u_{n}{ }^{6}\right) \psi_{j_{x}}(t)+\int u_{n_{x}}^{2} \psi_{j_{x x x}}(t) .
\end{aligned}
$$

Again $m_{j}^{\prime}(t) \geq \sigma_{0}$ and $\left|m_{j}^{\prime}(t)\right| \leq c_{N}$, so that $\int u_{n_{x}}^{2} \psi_{j x x x}(t)-\frac{\sigma_{0}}{4} \int u_{n_{x}}^{2} \psi_{j_{x}}(t) \geq 0$ and

$$
\begin{aligned}
\frac{d}{d t} \int\left(u_{n x}^{2}-\frac{u_{n}^{6}}{3}\right) \psi_{j}(t) & \geq \frac{3}{4} \sigma_{0} \int u_{n x}^{2}\left|\psi_{j_{x}}(t)\right| \\
& -\int\left(10 u_{n_{x}}^{2}\left|u_{n}\right|^{4}-\frac{c_{N}}{3}\left|u_{n}\right|^{6}\right)\left|\psi_{j_{x}}(t)\right| .
\end{aligned}
$$


To bound $10 \int u_{n_{x}}^{2}\left|u_{n}\right|^{p-1}\left|\psi_{j_{x}}(t)\right|$, we proceed like before and get

$$
10 \int u_{n_{x}}^{2}\left|u_{n}\right|^{4}\left|\psi_{j_{x}}(t)\right| \geq-\frac{\sigma_{0}}{4} \int\left|u_{n_{x}}^{2}\right| \psi_{j_{x}}(t)-C e^{-\frac{\sigma_{0} \sqrt{\sigma_{0}}}{4} t} .
$$

However for $\frac{c_{N}}{6} \int u_{n}{ }^{6}\left|\psi_{j_{x}}(t)\right|$, some $L^{2}$ norm is needed (which is why we introduced $F_{j}$, as in [11]). Choosing $\varepsilon_{1}$ small enough and $T_{1}$ large enough, we can improve (33) to $\sigma_{0} / 400$, and so obtain

$$
\frac{c_{N}}{3} \int u_{n}{ }^{6} \geq-\frac{\sigma_{0}}{400} \int u_{n}{ }^{2}\left|\psi_{j_{x}}(t)\right|-C e^{-\frac{\sigma_{0} \sqrt{\sigma_{0}}}{4} t} .
$$

Now adding up (35) and 1/100.(34), and using (36) and (37), we get

$$
\frac{d}{d t} \int\left(u_{n_{x}}^{2}-\frac{1}{3} u_{n}{ }^{6}+\frac{1}{200} u_{n}^{2}\right) \psi_{j}(t) \geq \frac{\sigma_{0}}{2} \int u_{n_{x}}^{2}\left|\psi_{x}(t)\right|-C e^{-\frac{\sigma_{0} \sqrt{\sigma_{0}}}{4} t} .
$$

And the estimate on $F_{j}$ comes by integration between $t$ and $S_{n}$.

Remark 5. Notice that it is possible to obtain almost monotonicity properties (on the left) related to other quantities than mass and energy. However, these are related to conservation laws : thus they translate to monotonicity properties on the right, and this is specially interesting and useful.

We can now conclude the proof of Proposition 2.

Proof of Proposition 2. We do some estimates on $\tilde{w}_{n}(t)$ first. The key point is the following resummation argument, which will allow us to use the monotonicity property. We compute

$$
\begin{aligned}
& \sum_{j=1}^{N} \frac{1}{\gamma_{j}^{2}(t)}\left(E_{j}+\frac{\gamma_{j}(t)}{2} M_{j}\right)=\sum_{j=1}^{N-1}\left(\left(\frac{1}{\gamma_{j}^{2}(t)}-\frac{1}{\gamma_{j+1}^{2}(t)}\right) \sum_{k=1}^{j} F_{k}\right) \\
&+\sum_{j=1}^{N-1}\left(\frac{1}{2}\left(\frac{1}{\gamma_{j}(t)}-\frac{1}{\gamma_{j+1}(t)}\right)\left(1-\frac{\sigma_{0}}{50}\left(\frac{1}{\gamma_{j}(t)}+\frac{1}{\gamma_{j+1}(t)}\right)\right) \sum_{k=1}^{j} M_{k}\right) \\
&+\frac{1}{\gamma_{N}^{2}(t)} \sum_{k=1}^{N} F_{k}+\frac{1}{2 \gamma_{N}(t)}\left(1-\frac{\sigma_{0}}{50 c_{N}}\right) \sum_{j=1}^{N} M_{k} .
\end{aligned}
$$

All the terms in the right hand side are positives, so that we can apply Lemma 5 :

$$
\begin{array}{r}
\sum_{j=1}^{N} \frac{1}{\gamma_{j}^{2}(t)}\left(E_{j}(t)+\frac{\gamma_{j}(t)}{2} M_{j}(t)\right)-\sum_{j=1}^{N} \frac{1}{\gamma_{j}^{2}(t)}\left(E_{j}\left(S_{n}\right)+\frac{\gamma_{j}(t)}{2} M_{j}\left(S_{n}\right)\right) \\
\leq C e^{-\frac{\sigma_{0} \sqrt{\sigma_{0}}}{4} t} .
\end{array}
$$

Now we use fact 3 . of Lemma 4 at time $t$ and at time $S_{n}$ (remind that $\mid \gamma_{j}(t)-$ $c_{j} \mid \leq C \varepsilon_{0}$, so that $\left.c_{N}+\varepsilon_{0} \geq \gamma_{j}(t) \geq \sigma_{0}\right)$ :

$$
\sum_{j=1}^{N} \frac{1}{\gamma_{j}^{2}(t)} H_{j}(t)
$$




$$
\begin{aligned}
& \leq C e^{-\frac{\sigma_{0} \sqrt{\sigma_{0}}}{2} t}+C \varepsilon_{0} \int \tilde{v_{n}}{ }^{2}(t) \sum_{j=1}^{N} \phi_{j}(t)+C \varepsilon_{0} \int{\tilde{v_{n}}}^{2}\left(S_{n}\right) \sum_{j=1}^{N} \phi_{j}\left(S_{n}\right) \\
& \leq C e^{-\frac{\sigma_{0} \sqrt{\sigma_{0}}}{2} t}+C \varepsilon_{0}\left\|\tilde{v_{n}}(t)\right\|_{L^{2}\left(1-\psi_{0}(t)\right)}^{2}+C \varepsilon_{0}\left\|U\left(S_{n}\right) V\right\|_{L^{2}\left(1-\psi_{0}\left(S_{n}\right)\right)}^{2} .
\end{aligned}
$$

By Proposition 4, we have that for $j=1, \ldots, N$,

$$
H_{j}(t) \geq \lambda_{1} \int\left({\tilde{v_{n}}}^{2}(t)+\tilde{v}_{x}^{2}(t)\right) \phi_{j}(t)-\frac{1}{\lambda_{1}}\left(\left(\int \tilde{v}(t) \tilde{R}_{j}^{3}\right)^{2}+\left(\int \tilde{v}(t) \tilde{R}_{j_{x}}\right)^{2}\right) .
$$

So that if we sum up those $N$ inequalities, there exists $\lambda_{0}>0$ neither depending on $\sigma_{0}$ nor $\varepsilon_{0}$ ) such that

$$
\begin{aligned}
& \sum_{j=1}^{N} \frac{1}{\gamma_{j}^{2}(t)} H_{j}(t) \\
& \left.\geq \lambda_{0} \| \tilde{v_{n}}(t)\right) \|_{H^{1}\left(1-\psi_{0}(t)\right)}^{2}-\frac{1}{\lambda_{0}} \sum_{j=1}^{N}\left(\left(\int \tilde{v_{n}}(t) \tilde{R}_{j}^{3}(t)\right)^{2}+\left(\int \tilde{v_{n}}(t) \tilde{R}_{j_{x}}(t)\right)^{2}\right) \\
& \left.\geq \lambda_{0} \| \tilde{v_{n}}(t)\right) \|_{H^{1}\left(1-\psi_{0}(t)\right)}^{2}-\frac{1}{\lambda_{0}} \sum_{j=1}^{N}\left(\left(\int U(t) V \tilde{R}_{j}^{3}\right)^{2}+\left(\int U(t) V \tilde{R}_{j_{x}}\right)^{2}\right) \\
& \left.\geq \lambda_{0} \|{\tilde{v_{n}}}(t)\right)\left\|_{H^{1}\left(1-\psi_{0}(t)\right)}^{2}-\frac{C}{\lambda_{0}}\right\| U(t) V \|_{L^{2}\left(1-\psi_{0}(t)\right)}^{2} .
\end{aligned}
$$

Combining (39) and (38), provided that $\varepsilon_{0}$ is small enough so that $C_{3} \varepsilon_{0}<\lambda_{0} / 2$, we deduce

$\left.\frac{1}{C} \| \tilde{v_{n}}(t)\right)\left\|_{H^{1}\left(1-\psi_{0}(t)\right)}^{2} \leq e^{-\frac{\sigma_{0} \sqrt{\sigma_{0}}}{4} t}+\right\| U(t) V\left\|_{L^{2}\left(1-\psi_{0}(t)\right)}^{2}+\right\| U\left(S_{n}\right) V \|_{L^{2}\left(1-\psi_{0}\left(S_{n}\right)\right)}^{2}$.

We will only use the obtained bound on $\left.\| \tilde{v_{n}}(t)\right) \|_{L^{2}\left(1-\psi_{0}(t)\right)}$. Recall $\tilde{v_{n}}(t)=$ $\tilde{w}_{n}(t)+U(t) V$, thus

$$
\begin{aligned}
& \left.\left.\| \tilde{w}_{n}(t)\right)\left\|_{L^{2}\left(1-\psi_{0}(t)\right)}^{2} \leq 2\right\| \tilde{v_{n}}(t)\right)\left\|_{L^{2}\left(1-\psi_{0}(t)\right)}^{2}+2\right\| U(t) V \|_{L^{2}\left(1-\psi_{0}(t)\right)}^{2} \\
& \leq C e^{-\frac{\sigma_{0} \sqrt{\sigma_{0}}}{4} t}+C\|U(t) V\|_{L^{2}\left(1-\psi_{0}(t)\right)}^{2}+C\left\|U\left(S_{n}\right) V\right\|_{L^{2}\left(1-\psi_{0}\left(S_{n}\right)\right)}^{2} .
\end{aligned}
$$

Relying on estimate (40), we only need to go back to $w_{n}(t)=\tilde{w}_{n}(t)+R(t)-$ $\tilde{R}(t)$. As we noted in (32),

$$
\begin{aligned}
\left\|w_{n}(t)\right\|_{L^{2}\left(1-\psi_{0}(t)\right)} \leq & \left.\|R(t)-\tilde{R}(t)\|_{L^{2}}+\| \tilde{w}_{n}(t)\right) \|_{L^{2}\left(1-\psi_{0}(t)\right)} \\
\leq & C \sum_{k=1}^{N}\left|y_{j}(t)-x_{j}-c_{j} t\right|+\left|\gamma_{j}(t)-c_{j}\right|+C e^{-\frac{\sigma_{0} \sqrt{\sigma_{0}}}{4} t} \\
& +C\|U(t) V\|_{L^{2}\left(1-\psi_{0}(t)\right)}+C\|U(t) V\|_{L^{2}\left(1-\psi_{0}\left(S_{n}\right)\right)}
\end{aligned}
$$

Now, using the $L_{\text {loc }}^{2}$ estimate of lemma 3 , and (40) :

$$
\begin{aligned}
& \left|y_{j}^{\prime}(t)-c_{j}\right|+\left|\gamma_{j}^{\prime}(t)\right| \\
& \quad \leq C e^{-\frac{\sigma_{0} \sqrt{\sigma_{0}}}{4} t}+C\|U(t) V\|_{L^{2}\left(1-\psi_{0}(t)\right)}+C\left(\int{\tilde{w_{n}}}^{2}(t) e^{-\sqrt{\sigma_{0}}\left|x-c_{j} t\right|}\right)^{1 / 2}
\end{aligned}
$$




$$
\leq C e^{-\frac{\sigma_{0} \sqrt{\sigma_{0}}}{8} t}+C\|U(t) V\|_{L^{2}\left(1-\psi_{0}(t)\right)}+C\left\|U\left(S_{n}\right) V\right\|_{L^{2}\left(1-\psi_{0}\left(S_{n}\right)\right)} .
$$

Let us integrate this between $t$ and $S_{n}$. Remind the initial conditions $y_{j}\left(S_{n}\right)=$ $x_{j}+c_{j} S_{n}, \gamma_{j}\left(S_{n}\right)=c_{j}$, we obtain

$$
\begin{aligned}
\left|y_{j}(t)-x_{j}-c_{j} t\right|+\left|\gamma_{j}(t)-c_{j}\right| \leq & C e^{-\frac{\sigma_{0} \sqrt{\sigma_{0}}}{4} t}+C \int_{t}^{S_{n}}\|U(t) V\|_{L^{2}\left(1-\psi_{0}(t)\right)} d t \\
& +C\left(S_{n}-t\right)\left\|U\left(S_{n}\right) V\right\|_{L^{2}\left(1-\psi_{0}\left(S_{n}\right)\right) .}
\end{aligned}
$$

This, together with (41), concludes the proof of Proposition 2.

\section{Control of the interaction of $w_{n}$ with $U(t) V$ : the linear theory}

This section is devoted to the proof of Proposition 3.

However, let us first link Proposition 2, Proposition 3, Lemma 2 and Proposition 1' together. We compute the decay we obtained on $\left\|w_{n}(t)\right\|_{H^{1}\left(1-\psi_{0}(t)\right)}$.

$\left(1+x_{+}\right)^{2+\delta_{0}} V \in H^{1}$, so that from Lemma 2 ,

$$
\|U(t) V\|_{L^{2}\left(1-\psi_{0}(t)\right)} \leq \frac{C}{t^{2+\delta_{0}}} .
$$

From Proposition 2, we can then conclude that

$$
\left\|w_{n}(t)\right\|_{L^{2}\left(1-\psi_{0}(t)\right)} \leq \frac{C}{t^{1+\delta_{0}}} .
$$

This ensures that the assumptions of Proposition 3 are fulfilled.

Thus, Proposition 1' follows from Proposition 2, Lemma 2 and Proposition 3.

\subsection{Preliminary lemmas}

First recall the fundamental linear estimate.

Lemma 6 ([7]). Let $f: \mathbb{R} \times \mathbb{R} \rightarrow \mathbb{R}$ and $B \in \mathbb{R}$. The following inequalities hold, as long as the right-hand side is bounded:

$$
\begin{aligned}
&\left\|\partial_{x} \int_{\tau}^{B} U(t-s) f(s, x) d s\right\|_{L_{x}^{5} L_{\tau}^{10}(\tau \in[t, B])} \leq\|f(\tau, x)\|_{L_{x}^{1} L_{\tau}^{2}(\tau \in[t, B])}, \\
& \sup _{\tau \in[t, B]}\left\|\partial_{x} \int_{\tau}^{B} U(t-s) f(s, x) d s\right\|_{L_{x}^{2}} \leq\|f(\tau, x)\|_{L_{x}^{1} L_{\tau}^{2}(\tau \in[t, B])} .
\end{aligned}
$$

Proof. In [7], the proof of the first estimate is done without restriction in time, that is

$$
\left\|\partial_{x} \int_{\tau}^{B} U(t-s) f(s) d s\right\|_{L_{x}^{5} L_{\tau}^{10}} \leq\|f\|_{L_{x}^{1} L_{\tau}^{2}} .
$$

Now as $s \in[\tau, B] \subset[t, B]$, we get

$$
\left\|\partial_{x} \int_{\tau}^{B} U(t-s) f(s) d s\right\|_{L_{x}^{5} L_{\tau}^{10}(\tau \in[t, B])}
$$




$$
\begin{aligned}
& =\left\|\partial_{x} \int_{\tau}^{B} U(t-s)\left(f(s) \mathbb{1}_{s \in[\tau, B]}\right) d s\right\|_{L_{x}^{5} L^{10}(\tau \in[t, B])} \\
& \leq\left\|\partial_{x} \int_{\tau}^{B} U(t-s)\left(f(s) \mathbb{1}_{s \in[\tau, B]}\right) d s\right\|_{L_{x}^{5} L^{10}} \\
& \leq\left\|f(s) \mathbb{1}_{s \in[\tau, B]}\right\|_{L_{x}^{1} L_{t}^{2}}=\|f\|_{L_{x}^{1} L_{\tau}^{2}(\tau \in[t, B])} .
\end{aligned}
$$

The proof of the second estimate with no restriction on time is done in $[6]$ : the restricted one is done analogously.

Now, let us prove a lemma which will handle the interference of the solitons when we will control the interaction of $w_{n}$ with the linear term $U(t) V$.

Lemma 7 (Weak interference of solitons). Let $A \geq 1, B \geq A, \delta_{0}>0$, and $f:[A, B] \times \mathbb{R} \rightarrow \mathbb{R}$. Suppose that

$$
f \in L_{x}^{5} L_{t}^{10}(t \in[A, B]) \quad \text { and } \quad \forall t \in[A, B], \quad\|f(t)\|_{L^{2}\left(1-\psi_{0}(t)\right)} \leq \frac{C}{t^{1+\delta_{0}}} .
$$

Then there exists $C$ (independent of $A$ and $B$ ) such that

$$
\forall t \in[A, B], \quad\|f R\|_{L_{x}^{1} L_{\tau}^{2}(\tau \in[t, B])} \leq C e^{-\frac{\sigma_{0} \sqrt{\sigma_{0}}}{4} t}\|f\|_{L_{x}^{5} L_{\tau}^{10}(\tau \in[t, B])}+\frac{C}{t^{\delta_{0}}} .
$$

Remark 6. Observe that this result is almost optimal with respect to the decay rate required on $\|f(t)\|_{L^{2}\left(1-\psi_{0}(t)\right)}$. Indeed, suppose that $f(t, x)=\frac{1}{t^{\alpha}} Q(x-t)$. Then

$$
\begin{aligned}
\|f(\tau, x) Q(x-t)\|_{L_{x}^{1} L_{\tau}^{2}(\tau \in[t, B])} & =\int\left(\int_{\tau \in[t, B]} Q^{3}(x-t) \frac{d \tau}{\tau^{2 \alpha}}\right)^{1 / 2} d x \\
& \sim \int_{x \in[t, B]} \frac{d x}{x^{\alpha}} \sim \frac{C}{t^{1-\alpha}} .
\end{aligned}
$$

Thus, in order to have a decay estimate, we have to impose $\alpha>1$, and we lose one order of decay.

Proof. First notice that it is enough to obtain the result for a single soliton. Indeed, to conclude for the $N$-soliton case, it suffices to see

$$
\|f R\|_{L_{x}^{1} L_{\tau}^{2}(\tau \in[t, B])} \leq \sum_{j=1}^{N}\left\|f R_{j}\right\|_{L_{x}^{1} L_{\tau}^{2}(\tau \in[t, B])} .
$$

The idea is to split the double integral into two pieces, depending whether $\left|x-c_{j} \tau-x_{j}\right| \geq\left(x-x_{j}\right) / 2$ or not. Denote

$$
A(x)=\left\{\tau: \tau \in[t, B],\left|x-c_{j} \tau-x_{j}\right| \geq \frac{\left|x-x_{j}\right|}{2}\right\}, \text { and } B(x)=[t, B] \backslash A(x) .
$$

Then

$$
\left\|f R_{j}(s)\right\|_{L_{x}^{1} L_{\tau}^{2}(\tau \in[t, B])}=\int_{x}\left(\int_{\tau \in[t, B]}\left|f R_{j}\right|^{2}(\tau, x) d \tau\right)^{1 / 2} d x
$$




$$
\begin{aligned}
= & \int_{x}\left(\int_{\tau \in A(x)}\left|f R_{j}\right|^{2}(\tau, x) d \tau\right)^{1 / 2} d x \\
& +\int_{x}\left(\int_{\tau \in B(x)}\left|f R_{j}\right|^{2}(\tau, x) d \tau\right)^{1 / 2} d x \\
= & I+I I .
\end{aligned}
$$

We estimate separately $I$ and $I I$.

For $I$, we are "away" from the soliton, and we use its decay to go from $L_{x}^{1} L_{t}^{2}$ to $L_{x}^{5} L_{t}^{10}$, with an exponentially small constant. Recall $R_{j}(t, x)=Q_{c_{j}}\left(x-c_{j} t-x_{j}\right)$. Remark that as $Q(y)$ is even and decreasing (to 0 ) for $y \geq 0$,

$$
\sup _{\tau \in A(x)}\left|R_{j}(\tau, x)\right|=Q_{c_{j}}\left(\frac{x-x_{j}}{2}\right) .
$$

So that using Hölder's inequality in the $\tau$ integral with exponents $\frac{1}{2}=\frac{1}{10}+\frac{2}{5}$, we get

$$
\begin{array}{r}
I \leq \int_{x} Q_{c_{j}}^{1 / 2}\left(\frac{x-x_{j}}{2}\right)\left(\int_{\tau \in A(x)}|f|^{2}(\tau, x) Q_{c_{j}}\left(x-c_{j} \tau-x_{j}\right) d \tau\right)^{\frac{1}{2}} d x \\
\leq \int_{x} Q_{c_{j}}^{1 / 2}\left(\frac{x-x_{j}}{2}\right)\left(\int_{\tau \in A(x)}|f(\tau, x)|^{10} Q_{c_{j}}\left(x-c_{j} \tau-x_{j}\right) d \tau\right)^{\frac{1}{10}} \\
\times\left(\int_{\tau \in A(x)} Q_{c_{j}}\left(x-c_{j} \tau-x_{j}\right) d \tau\right)^{\frac{2}{5}} d x .
\end{array}
$$

Now let $(\tau, x)$ such that $\tau \in A(x)$. We claim that $\left|x-c_{j} \tau-x_{j}\right| \geq \frac{c_{j}}{3} \tau \geq \frac{c_{j}}{3} t$.

Indeed : first suppose $x-x_{j} \geq c_{j} \tau$. As $\tau \geq t \geq A \geq 0, x-x_{j} \geq 0$, and we have $x-c_{j} \tau-x_{j} \geq\left(x-x_{i}\right) / 2$. Thus $x-x_{j} \geq 2 c_{j} \tau$, so that $\left|x-c_{j} \tau-x_{j}\right|=$ $x-c_{j} \tau-x_{j} \geq c_{j} \tau$.

Else if $x-x_{j} \leq c_{j} \tau$ : if $x-x_{j} \leq 0$, as $\tau \geq 0$, we get $\left|x-c_{j} \tau-x_{j}\right|=$ $c_{j} \tau-\left(x-x_{j}\right) \geq c_{j} \tau$. Else if $x-x_{j} \geq 0$, then $c_{j} \tau-\left(x-x_{j}\right) \geq\left(x-x_{j}\right) / 2$, so that $\frac{2}{3} c_{j} \tau \geq\left(x-x_{j}\right)$. Thus, $c_{j} \tau-\left(x-x_{j}\right) \geq \frac{1}{3} c_{j} \tau$. This proves our claim.

And we get (with $y=x-c_{j} t-x_{j}, d y=c_{j} d \tau$ )

$$
\begin{aligned}
\left(\int_{\tau \in A(x)} Q_{c_{j}}\left(x-c_{j} \tau-x_{j}\right) d \tau\right)^{2 / 5} & \leq\left(\int_{|y| \geq c_{j} / 3 t} Q_{c_{j}}(y) \frac{d y}{c_{j}}\right)^{2 / 5} \\
& \leq C e^{-\frac{2 c_{j} \sqrt{c_{j}}}{15} t} .
\end{aligned}
$$

Applying again Hölder's inequality (in the $x$ integral) with exponent $1=\frac{4}{5}+\frac{1}{5}$, we can thus estimate

$$
\begin{aligned}
& I \leq C e^{-\frac{2 c_{j} \sqrt{c_{j}}}{15} t} \int_{x} Q_{c_{j}}^{1 / 2}\left(\frac{x-x_{j}}{2}\right) \\
& \quad \times\left(\int_{\tau \in A(x)}|f|^{10}(\tau, x) Q_{c_{j}}\left(x-c_{j} \tau-x_{j}\right) d \tau\right)^{\frac{1}{10}} d x \\
& \leq C e^{-\frac{2 c_{j} \sqrt{c_{j}}}{15} t}\left(\int_{x} Q_{c_{j}}^{5 / 8}\left(\frac{x-x_{j}}{2}\right) d x\right)^{4 / 5}
\end{aligned}
$$




$$
\begin{aligned}
& \quad \times\left(\int_{x}\left(\int_{\tau \in A(x)}|f|^{10}(\tau, x) Q_{c_{j}}\left(x-c_{j} \tau-x_{j}\right) d \tau\right)^{1 / 2} d x\right)^{1 / 5} \\
& \leq C e^{-\frac{2 c_{j} \sqrt{c_{j}}}{15} t}\left\|Q_{c_{j}}^{1 / 2}\right\|_{L^{5 / 4}}\left\|f R_{j}^{1 / 10}\right\|_{L_{x}^{5} L_{\tau}^{10}(\tau \in[t, B])} \\
& \leq C e^{-\frac{\sigma_{0} \sqrt{\sigma_{0}}}{4} t}\|f\|_{L_{x}^{5} L_{\tau}^{10}(\tau \in[t, B]) .}
\end{aligned}
$$

For $I I$, we have the full bump of the soliton, but $x-x_{j} \geq \frac{1}{3} c_{j} \tau$, so we can use our decay on the right. This decay is in $L_{t}^{\infty} L_{x}^{2}$, so we have to interchange integrals : we will decompose the $x$ integral in intervals $x \sim 2^{j}$, so that when applying the Cauchy-Schwarz inequality (to have $L_{x}^{2} L_{t}^{2}$, and then apply the Fubini-Tonelli Theorem), we don't pay too high a price.

Notice that for $(\tau, x)$ such that $\tau \in B(x),\left|\left(x-x_{j}\right)-c_{j} \tau\right| \leq\left|x-x_{j}\right| / 2$, so that $x-x_{j} \geq 0$ because $\tau \geq 0$. This implies that we can restrict ourselves to $x \geq x_{j}$ in the integral in $x$. Let $L_{p}=2^{p}-1+x_{j}$. Then

$$
I I=\sum_{p \in \mathbb{N}} \int_{x=L_{p}}^{L_{p+1}}\left(\int_{\tau \in B(x)}\left|f R_{j}\right|^{2}(\tau, x) d \tau\right)^{1 / 2} d x,
$$

and by Cauchy-Schwarz inequality,

$$
\leq \sum_{p \in \mathbb{N}} 2^{p / 2}\left(\int_{x=L_{p}}^{L_{p+1}} \int_{\tau \in B(x)}\left|f R_{j}\right|^{2}(\tau, x) d \tau d x\right)^{1 / 2} .
$$

Now let $(x, \tau)$ such that $x \in\left[L_{p}, L_{p+1}\right.$ and $\tau \in B(x)$. Then $\left|\left(x-x_{j}\right)-c_{j} t\right| \leq$ $\left|x-x_{j}\right| / 2$ and $x-x_{j} \geq 0$ implies that

$c_{j} \tau \in\left[\left(x-x_{j}\right)-\left(x-x_{j}\right) / 2,\left(x-x_{j}\right)+\left(x-x_{j}\right) / 2\right]=\left[\left(x-x_{j}\right) / 2,3\left(x-x_{j}\right) / 2\right]$, so that $\tau \in\left[\left(2^{p}-1\right) /\left(2 c_{j}\right), 3\left(2^{p+1}-1\right) /\left(2 c_{j}\right)\right]$ and of course $\tau \in[t, B]$. This means that :

$$
\begin{aligned}
\left\{(\tau, x): x \in\left[L_{p}, L_{p+1}\right], \tau\right. & \in B(x)\} \\
& \subset\left[L_{p}, L_{p+1}\right] \times\left(\left[\frac{2^{p}-1}{2 c_{j}}, \frac{3\left(2^{p+1}-1\right)}{2 c_{j}}\right] \cap[t, B]\right),
\end{aligned}
$$

which is a rectangle: thus we can interchange integrals.

$$
\begin{aligned}
\int_{x=L_{p}}^{L_{p+1}} \int_{\tau \in B(x)}\left|f R_{j}\right|^{2}(\tau, x) d \tau d x & \leq \int_{x=L_{p}}^{L_{p+1}} \int_{\substack{\tau \in\left[\frac{2^{p}-1}{2 c_{j}}, \frac{3\left(2^{p+1}-1\right)}{2 c_{j}}\right]\\
}}\left|f R_{j}\right|^{2}(\tau, x) d \tau d x \\
\substack{\tau \in\left[\tau \in[t, B], \tau \in\left[\frac{2^{p}-1}{2 c_{j}}, \frac{\left.3(2)^{p+1}-1\right)}{2 c_{j}}\right]\right.} & \int_{x=L_{p}}^{L_{p+1}}\left|f R_{j}\right|^{2}(\tau, x) d x d \tau .
\end{aligned}
$$

Define $K_{1}$ the maximal index such that $\left(2^{K_{1}}-1\right) /\left(2 c_{j}\right) \leq t$, and $K_{2}$ the maximal index such that $\left(2^{K_{2}}-1\right) /\left(2 c_{j}\right) \leq B$. We can now use our decay estimate on $\|f(t)\|_{L^{2}\left(1-\psi_{0}(t)\right)}$ :

$$
I I \leq \sum_{p=K_{1}}^{K_{2}} 2^{p / 2}\left(\int_{\tau \in\left[\frac{2^{p}-1}{2 c_{j}}, \frac{3\left(2^{p+1}-1\right)}{2 c_{j}}\right]} \int_{x}\left|f R_{j}\right|^{2}(\tau, x) d x d \tau\right)^{1 / 2}
$$




$$
\begin{gathered}
\left.+2^{\left(K_{2}+1\right) / 2} \int_{\tau: \tau \in\left[\frac{\left.2_{2-1}-B\right]}{2 c_{j}}, B\right]} \int_{x}\left|f R_{j}\right|^{2}(\tau, x) d x d \tau\right)^{1 / 2} \\
\leq \sum_{p=K_{1}}^{K_{2}} 2^{p / 2}\left(\int_{\tau=\frac{2^{p}-1}{2 c_{j}}}^{\frac{3\left(2^{p+1}-1\right)}{2 c_{j}}}\|f(\tau)\|_{L^{2}\left(1-\psi_{0}(\tau)\right)}^{2} d \tau\right)^{1 / 2} \\
\left.+2^{\left(K_{2}+1\right) / 2} \int_{\tau=\frac{2^{K} K_{2}-1}{2 c_{j}}}^{B}\|f(\tau)\|_{L^{2}\left(1-\psi_{0}(\tau)\right)}^{2} d \tau\right)^{1 / 2} \\
\leq C \sum_{p=K_{1}}^{K_{2}+1} 2^{p / 2}\left(\int_{\tau=\frac{2^{p}-1}{2 c_{j}}}^{\frac{3\left(2^{p+1}-1\right)}{2 c^{2}}} \frac{d \tau}{\tau^{2+2 \delta_{0}}}\right)^{1 / 2} \\
\leq C \sum_{p=K_{1}}^{K_{2}+1} 2^{p / 2}\left(2^{p-1}-1\right)^{-1 / 2-\delta_{0}} .
\end{gathered}
$$

As $2^{p / 2}\left(2^{p-1}-1\right)^{-1 / 2-\delta_{0}} \leq C 2^{-p \delta_{0}}$ and $\left(2^{K_{1}}-1\right) /\left(2 c_{j}\right) \geq t / 2$, which means $C 2^{K_{1}} \geq t$, we get

$$
I I \leq C \sum_{p=K_{1}}^{K_{2}+1} 2^{-p \delta_{0}} \leq C 2^{-K_{1} \delta_{0}} \leq C t^{-\delta_{0}} .
$$

Summing up (47) and (48) yields the result (46).

Lemmas 6 and 7 will be used with $f=w_{n}+U(t) V, A=T_{0}$ and $B=S_{n}$.

\subsection{Proof of Proposition 3}

Proof of Proposition 3. From (9) and Duhamel formula, $w_{n}(t)$ satisfies the following integral formulation :

$w_{n}\left(S_{n}\right)=U\left(S_{n}-t\right) w_{n}(t)+\partial_{x} \int_{t}^{S_{n}}\left(\left(w_{n}(\tau)+U(\tau) V+R(\tau)\right)^{5}-\sum_{j} R_{j}^{5}(\tau)\right) d \tau$.

Compose by $U\left(t-S_{n}\right)$, as recall that $w_{n}\left(S_{n}\right)=0$, so that

$$
\begin{array}{r}
w_{n}(t)=-\partial_{x} \int_{t}^{S_{n}} U(t-\tau)\left(\left(w_{n}(\tau)+U(\tau) V+R(\tau)\right)^{5}-\sum_{j} R_{j}^{5}(\tau)\right) d \tau \\
=-\sum_{k=1}^{5} C_{5}^{k} \partial_{x} \int_{t}^{S_{n}} U(t-\tau)\left(\left(w_{n}(\tau)+U(\tau) V\right)^{k} R^{5-k}(\tau)\right) d \tau \\
-\partial_{x} \int_{t}^{S_{n}} U(t-\tau)\left(R^{5}(\tau)-\sum_{j=1}^{N} R_{j}^{5}(\tau)\right) d s .
\end{array}
$$

We now use the $L_{x}^{5} L_{t}^{10}$ setting of [7]. According to (49), with estimates (44) and (45), we have

$$
\begin{gathered}
\left\|w_{n}(\tau, x)\right\|_{C^{0}\left(\left[t, S_{n}\right], L_{x}^{2}\right)}+\left\|w_{n}(\tau, x)\right\|_{L_{x}^{5} L_{\tau}^{10}\left(\tau \in\left[\tau, S_{n}\right]\right)} \\
\leq\left\|\left(w_{n}+U(t) V\right)^{5}\right\|_{L_{x}^{1} L_{\tau}^{2}\left(\tau \in\left[t, S_{n}\right]\right)}
\end{gathered}
$$




$$
\begin{aligned}
& +C \sum_{k=1}^{4} C_{5}^{k}\left\|\left(w_{n}(\tau)+U(\tau) V\right)^{k} R^{5-k}(\tau)\right\|_{L_{x}^{1} L_{\tau}^{2}\left(\tau \in\left[t, S_{n}\right]\right)} \\
& +C\left\|R^{5}(\tau)-\sum_{j=1}^{N} R_{j}^{5}(\tau)\right\|_{L_{x}^{1} L_{\tau}^{2}\left(\tau \in\left[t, S_{n}\right]\right)}
\end{aligned}
$$

First consider the last term. Recall the simple inequality

$$
|z-a|+|z-b| \geq 2\left|z-\frac{a+b}{2}\right|+\frac{|a-b|}{2} .
$$

As $\left|R_{j}(t, x)\right| \leq C e^{-\frac{\sqrt{\sigma 0}}{2}\left|x-x_{j}-c_{j} t\right|}$, we get that for $i \neq j$,

$$
\left|R_{i}(t, x) R_{j}(t, x)\right| \leq C e^{-\sqrt{\sigma_{0}}\left|x-\frac{x_{i}+x_{j}}{2}-\frac{c_{i}+c_{j}}{2} t\right|} e^{-\frac{\sqrt{\sigma_{0}}}{4}\left|c_{i}-c_{j}\right| t} .
$$

As $\left|c_{j}-c_{j}\right| \geq 2 \sigma_{0}$, we obtain

$$
\left\|R^{5}(\tau, x)-\sum_{j=1}^{N} R_{j}^{5}(\tau, x)\right\|_{L_{x}^{1} L_{\tau}^{2}(\tau \geq t)} \leq C\left(\sigma_{0}\right) e^{-\frac{\sigma_{0} \sqrt{\sigma_{0}}}{2} t} .
$$

Now consider the purely linear interaction $(k=5)$, that is the first term in (50) :

$$
\begin{aligned}
\left\|\left(w_{n}(\tau)+U(\tau) V\right)^{5}\right\|_{L_{x}^{1} L_{\tau}^{2}\left(\tau \in\left[t, S_{n}\right]\right)} & =\left\|w_{n}(\tau)+U(\tau) V\right\|_{L_{x}^{5} L_{\tau}^{10}\left(\tau \in\left[t, S_{n}\right]\right)}^{5} \\
& \leq C\|w\|_{\mathcal{N}\left(\left[t, S_{n}\right]\right)}^{5}+C\|U(\tau) V\|_{L_{x}^{5} L_{\tau}^{10}(\tau \geq t)}^{5} .
\end{aligned}
$$

It remains to control in (50) the terms with an interaction between $w_{n}+U(t) V$ and the solitons.

From (42) and (43), Lemma 7 applies to all the remaining terms in (50) (i.e. $k=1,2,3,4)$, to give

$$
\begin{aligned}
& \left\|\left(w_{n}(\tau)+U(\tau) V\right)^{k} R^{5-k}(\tau)\right\|_{L_{x}^{1} L_{\tau}^{2}\left(\tau \in\left[t, S_{n}\right]\right)} \\
& \leq C e^{-\frac{\sigma_{0} \sqrt{\sigma_{0}}}{4} t}\left\|w_{n}(\tau)+U(\tau) V\right\|_{L_{x}^{5} L_{\tau}^{10}\left(\tau \in\left[t, S_{n}\right]\right)}+\frac{C}{t^{\delta_{0}}} \\
& \leq C e^{-\frac{\sigma_{0} \sqrt{\sigma_{0}}}{4} t}\left\|w_{n}\right\|_{\mathcal{N}\left(\left[t, S_{n}\right]\right)}+C e^{-\frac{\sigma_{0} \sqrt{\sigma_{0}}}{4}} t\|U(\tau) V\|_{L_{x}^{5} L_{\tau}^{10}(\tau \geq t)}+\frac{C}{t^{\delta_{0}}} .
\end{aligned}
$$

(recall that $\left\|w_{n}(t)\right\|_{H^{1}}$ is uniformly bounded, like $\|U(t) V\|_{H^{1}}$, so that $\| w_{n}(\tau)+$ $U(\tau) V \|_{L_{x, \tau}^{\infty}\left(\tau \in\left[I_{n}, S_{n}\right]\right)} \leq C$ uniform in $n$ ). Summing up (51), (52) and (53), and plugging it in (50), we obtain

$$
\begin{aligned}
\|w\|_{\mathcal{N}\left(\left[t, S_{n}\right]\right)}= & \left\|w_{n}(\tau, x)\right\|_{C^{0}\left(\left[t, S_{n}\right], L_{x}^{2}\right)}+\left\|w_{n}(\tau, x)\right\|_{L_{x}^{5} L_{\tau}^{10}\left(\tau \in\left[t, S_{n}\right]\right)} \\
\leq & C\left\|w_{n}\right\|_{\mathcal{N}\left(\left[t, S_{n}\right]\right.}^{5}+C e^{-\frac{\sigma_{0} \sqrt{\sigma 0}}{4} t} t\left\|w_{n}\right\|_{\mathcal{N}\left(\left[t, S_{n}\right]\right)} \\
& +C\|U(\tau) V\|_{L_{x}^{5} L_{\tau}^{10}(\tau \geq t)}^{5}+C e^{-\frac{\sigma_{0} \sqrt{\sigma_{0}}}{2} t} t\|U(\tau) V\|_{L_{x}^{5} L_{\tau}^{10}(\tau \geq t)}+\frac{C}{t^{\delta_{0}}}
\end{aligned}
$$


Then for $\varepsilon_{0}$ small enough so that $C \varepsilon_{0}^{4} \leq 1 / 3$, and $T_{0}$ large enough so that $C e^{-\frac{\sigma_{0} \sqrt{\sigma_{0}}}{4}} T_{0} \leq 1 / 3$, we get that

$$
\forall t \in\left[I_{n}, S_{n}\right], \quad\left\|w_{n}\right\|_{\mathcal{N}\left(\left[t, S_{n}\right]\right)} \leq \eta(t),
$$

where

$$
\eta(t)=C\|U(\tau) V\|_{L_{x}^{5} L_{\tau}^{10}(\tau \geq t)}^{5}+C e^{-\frac{\sigma_{0} \sqrt{\sigma} 0}{4} t}\|U(\tau) V\|_{L_{x}^{5} L_{\tau}^{10}(\tau \geq t)}+\frac{C}{t^{\delta_{0}}}
$$

satisfies the conditions of Proposition 1'.

\section{Appendix}

We state a version of the implicit function theorem, to be used in the proof of Lemma 3.

Implicit function Theorem with parameter. Let $E, F, G, H$ be Banach spaces, and $f: E \times F \times G \rightarrow H$ a $C^{1}$ function. Let $U$ be an open set in $E$. We suppose there exist $C^{1}$ functions $x_{0}, y_{0}: U \rightarrow F, G$ such that for all $t \in U$, $f\left(t, x_{0}(t), y_{0}(t)\right)=0$, and that $\partial_{y} f\left(t, x_{0}(t), y_{0}(t)\right)$ is invertible.

Furthermore, we suppose that there exist $\delta_{0}>0, \eta_{0}>0$ such that

$$
\sup _{\substack{t \in U \\ x \in B\left(x_{0}(t), \delta_{0}\right) \\ y \in B\left(y_{0}(t), \eta_{0}\right)}}\left\|\partial_{y}^{-1} f\left(t, x_{0}(t), y_{0}(t)\right)\right\|\left\|\partial_{y} f\left(t, x_{0}(t), y_{0}(t)\right)-\partial_{y} f(t, x, y)\right\|=k<1,
$$

and

$$
\sup _{\substack{t \in U \\ x \in B\left(x_{0}(t), \delta_{0}\right) \\ y \in B\left(y_{0}(t), \eta_{0}\right)}}\left\|\partial_{y}^{-1} f\left(t, x_{0}(t), y_{0}(t)\right)\right\|\left\|\partial_{x} f(t, x, y)\right\|=C<\infty .
$$

Then there exist $\delta_{1}, \eta_{1}>0$ such that the following holds.

Define the tubular neighborhoods $V=\left\{(t, x) \mid t \in U, x \in B\left(\left(x_{0}(t), \delta_{1}\right)\right\}\right.$ and $W=\left\{(t, y) \mid t \in U, y \in B\left(\left(y_{0}(t), \eta_{1}\right)\right\}\right.$.

Then there exists a $C^{1}$ function $G: V \rightarrow W, G(t, x)=(t, g(t, x))$ such that

$\forall t, \forall x, y \in B\left(x_{0}(t), \eta_{1}\right) \times B\left(y_{0}(t), \eta_{1}\right), \quad f(t, x, y)=0 \Longleftrightarrow y=g(t, x)$.

Furthermore, $g(t, \cdot)$ is $(C+1) /(1-k)$-Lipschitz (in particular, we can choose $\left.\eta_{1} \leq(C+1) /(1-k) \cdot \delta_{1}\right)$.

Let us conclude with the proof of Proposition 4.

Proof of Proposition 4. See [17, Lemma 4] and [16, Appendix A], for the proof of a very similar result. The main idea is to use localization arguments on a definite positive operator. Indeed, recall that there exists $\lambda>0$ such that for all $v \in H^{1}$,

$$
\int\left(v_{x}^{2}-5 Q^{4} v^{2}+v^{2}\right) \geq \lambda\|v\|_{H^{1}}^{2}-\frac{1}{\lambda}\left(\left(\int v Q^{3}\right)^{2}+\left(\int v Q_{x}\right)^{2}\right) .
$$


By translation, and denoting

$$
\phi(t, x)=\psi\left(x-\left(m_{j-1} t-\gamma_{j}(t)\right)\right)-\psi\left(x-\left(m_{j} t-\gamma_{j}(t)\right)\right),
$$

we want to prove that for all $v \in H^{1}$,

$$
\int\left(v_{x}^{2}-5 Q^{4} v^{2}+v^{2}\right) \phi(t) \geq \lambda_{1} \int\left(v_{x}^{2}+v^{2}\right) \phi(t)-\frac{1}{\lambda_{1}}\left(\left(\int v Q^{3}\right)^{2}+\left(\int v Q_{x}\right)^{2}\right) .
$$

For simplicity, let us denote

$H(v, w)=\int\left(v_{x} w_{x}-5 Q^{4} v w+v w\right), \quad H_{\phi}(v, w)=\int\left(v_{x} w_{x}-5 Q^{4} v w+v w\right) \phi(t)$.

We begin by the following perturbation lemma concerning (56).

Lemma 8. There exists $\delta>0$ (depending only on $\lambda>0$ ) such that if $\left|\left(v \mid Q^{3}\right)\right|+$ $\left(v \mid Q_{x}\right) \mid \leq \delta\|v\|_{H^{1}}$, then

$$
H(v, v)=\int v_{x}^{2}-5 Q^{4} v^{2}+v^{2} \geq \frac{\lambda}{2} \int\left(v_{x}^{2}+v^{2}\right) .
$$

Proof. See [23], where an analoguous case is treated. Let us decompose in $L^{2}$ : $v=v_{1}+a Q^{3}+b Q_{x}=v_{1}+v_{2},\left(v_{1} \mid Q^{3}\right)=\left(v_{1} \mid Q_{x}\right)=0$, so that by hypothesis,

$$
|a|+|b| \leq \delta\|v\|_{H^{1}} .
$$

If $\delta<1 / 2$, we deduce that

$$
\frac{\sqrt{3}}{2}\|v\|_{H^{1}} \leq\left\|v_{1}\right\|_{H^{1}} \leq 2\|v\|_{H^{1}} .
$$

Now $H(v, v)=H\left(v_{1}, v_{1}\right)+H\left(v_{2}, v_{2}\right)+2 H\left(v_{1}, v_{2}\right)$. By $(56)$,

$$
H\left(v_{1}, v_{1}\right) \geq \lambda\left\|v_{1}\right\|_{H^{1}}^{2} \geq \frac{3 \lambda}{4}\|v\|_{H^{1}}^{2} .
$$

If $C \delta^{2} \leq \lambda / 8$ (where $C$ is the continuity norm of $H$ ),

$$
\left|H\left(v_{2}, v_{2}\right)\right| \leq C\left(|a|^{2}+|b|^{2}\right) \leq C \delta^{2}\|v\|_{H^{1}}^{2} \leq \frac{\lambda}{8}\|v\|_{H^{1}}^{2},
$$

and if $4 C \delta \leq \lambda / 8$,

$$
2\left|H\left(v_{1}, v_{2}\right)\right| \leq C\left\|v_{1}\right\|_{H^{1}}\left\|v_{2}\right\|_{H^{1}} \leq 4 C(|a|+|b|)\|v\|_{H^{1}} \leq 2 C \delta\|v\|_{H^{1}}^{2} \leq \frac{\lambda}{8}\|v\|_{H^{1}}^{2} .
$$

Finally :

$$
H(v, v) \geq\left(\frac{3}{4}-\frac{1}{8}-\frac{1}{8}\right) \lambda\|v\|_{H^{1}}^{2} \geq \frac{\lambda}{2}\|v\|_{H^{1}}^{2} .
$$

Lemma 9. There exists $\sigma_{1}>0$ such that the following is true. Given $\sigma_{0}<\sigma_{1}$, there exists $T_{3}=T_{3}(\sigma)$, such that if $\left(v, Q^{3}\right)=\left(v, Q_{x}\right)=0$, then

$$
H_{\phi}(v, v) \geq \frac{\lambda}{4} \int\left(v_{x}^{2}+v^{2}\right)(\phi) .
$$


Proof. Notice that $\left|(\phi)_{x}\right| \leq C \sqrt{\sigma_{0}} \phi$, the constant $C$ not depending on $t, x, \sigma_{0}$ (it is a computation involving $\psi$ ). Let $v \in H^{1}$ such that $\left(v \mid Q^{3}\right)=\left(v \mid Q_{x}\right)=0$. Now let us compute with $v \sqrt{\phi}$ :

$$
\begin{aligned}
H_{\phi}(v, v) & =\int\left(v_{x}^{2}-5 Q^{4} v^{2}+v^{2}\right) \phi \\
& \left.=\int(v \sqrt{\phi})_{x}^{2}-5 Q^{4}(v \sqrt{\phi})^{2}+(v \sqrt{\phi})^{2}\right)-\int v v_{x} \phi_{x}-\int v^{2}(\sqrt{\phi})_{x}^{2} \\
& =H(v \sqrt{\phi}, v \sqrt{\phi})-\int v v_{x} \phi_{x}-\int v^{2}(\sqrt{\phi})_{x}^{2} .
\end{aligned}
$$

Thanks to the orthogonality properties on $v$,

$$
\begin{aligned}
\left|\int v \sqrt{\phi} Q^{3}\right| & =\left|\int v(1-\sqrt{\phi}) Q^{3}\right| \leq C e^{-\frac{\sigma_{0} \sqrt{\sigma_{0}}}{4} t}\|v\|_{L^{2}} \text { and, } \\
\left|\int v \sqrt{\phi} Q_{x}\right| & =\left|\int v(1-\sqrt{\phi}) Q_{x}\right| \leq C e^{-\frac{\sigma_{0} \sqrt{\sigma} 0}{4} t}\|v\|_{L^{2}} .
\end{aligned}
$$

Thus, as soon as $t \geq T_{3}=T_{3}\left(\sigma_{0}\right)$ is large enough, we are in the setting of the previous lemma, and so

$$
H(v \sqrt{\phi}) \geq \frac{\lambda}{2}\|v \sqrt{\phi}\|_{H^{1}}^{2}=\frac{\lambda}{2}\left(\int\left(v_{x}^{2}+v^{2}\right) \phi+\int v v_{x} \phi_{x}+\int v^{2}(\sqrt{\phi})_{x}^{2}\right) .
$$

Thus :

$$
H_{\phi}(v, v) \geq \frac{\lambda}{2} \int\left(v_{x}^{2}+v^{2}\right) \phi+\left(\frac{\lambda}{2}-1\right) \int v v_{x} \phi_{x}+\left(\frac{\lambda}{2}-1\right) \int v^{2}(\sqrt{\phi})_{x}^{2} .
$$

We need to control the last two terms. First,

$$
\left|\int v v_{x} \phi_{x}\right| \leq C \sqrt{\sigma_{0}} \int\left|v v_{x}\right| \phi \leq C \sqrt{\sigma_{0}} \int\left(v_{x}^{2}+v^{2}\right) \phi,
$$

and in the same way, as $\left|(\sqrt{\phi})_{x}\right| \leq C \sqrt{\sigma_{0}} \sqrt{\phi}$,

$$
\int v^{2}(\sqrt{\phi})_{x}^{2} \leq C \sigma_{0} \int v^{2} \phi
$$

Choose $\sigma_{1} \leq \frac{1}{8 C} \frac{\lambda_{1}}{1-\lambda_{1} / 2}$, then for $t \geq T\left(\sigma_{0}\right)$ large enough, we get

$$
H_{\phi}(v, v) \geq \frac{\lambda}{4} \int\left(v_{x}^{2}+v^{2}\right)(\phi),
$$

as claimed.

We can now conclude the proof of Proposition 4. Let $v \in H^{1}$. Let us write the $L^{2}$ decomposition $v=v_{1}+a Q+b Q_{x}$, and develop :

$$
\begin{aligned}
H_{\phi}(v, v)=H_{\phi}\left(v_{1}, v_{1}\right)+2 a H_{\phi}\left(v_{1}, Q\right)+2 b H_{\phi}\left(v_{1}, Q_{x}\right) \\
+a^{2} H_{\phi}(Q, Q)+2 a b H_{\phi}\left(Q, Q_{x}\right)+b^{2} H_{\phi}\left(Q_{x}, Q_{x}\right) .
\end{aligned}
$$


By hypothesis, $H_{\phi}\left(v_{1}, v_{1}\right) \geq \lambda_{2} \int\left(v_{x}^{2}+v^{2}\right) \phi(t)$. Then, notice that $|a|=|(v \mid Q)| \leq$ $C\|v\|_{L^{2}}$ and $\left.|b| \leq\|v\|_{L^{2}}\right)$. Thus

$$
\left\|v_{1}\right\|_{H^{1}} \leq C\|v\|_{H^{1}} .
$$

Now $H\left(v_{1}, Q\right)=\left(v_{1}, \lambda_{Q} Q\right)=0$ as $Q$ (and $\left.Q_{x}\right)$ are eigenfunctions, and $H_{\phi}$ is symmetric : we deduce $H_{\phi}\left(v_{1}, a Q\right)=H_{1-\phi}\left(v_{1}, a Q\right)$ etc. And we get, by continuity of $H$,

$$
\left|H_{\phi}\left(v_{1}, a Q\right)\right|+\left|H_{\phi}\left(b Q_{x}, a Q\right)\right| \leq C\|v\|_{H^{1}}^{2}\left\|\left(\left|Q_{x}\right|+Q\right)(1-\phi(t))\right\|_{L^{\infty}} .
$$

And of course $\left\|\left(\left|Q_{x}\right|+Q\right)(1-\phi(t))\right\|_{L^{\infty}} \leq C e^{-\sigma_{0} \sqrt{\sigma_{0}} t}$. As $|\phi| \leq 1,\left|H_{\phi}(Q, Q)\right| \leq$ $\|Q\|_{H^{1}}^{2}$ and $\left|H_{\phi}\left(Q_{x}, Q_{x}\right)\right| \leq\left\|Q_{x}\right\|_{H^{1}}^{2}$. This gives

$$
H_{\phi}(v, v) \geq \lambda_{2} \int\left(v_{1 x}^{2}+v_{1}^{2}\right) \phi(t)-C e^{-\frac{\sigma_{0} \sqrt{\sigma_{0}}}{2} t}\|v\|_{H^{1}}^{2}-C\left(a^{2}+b^{2}\right) .
$$

Now,

$$
\begin{aligned}
\int\left(v_{1 x}^{2}+v_{1}^{2}\right) \phi(t) \geq & \int\left(v_{x}^{2}+v^{2}\right) \phi(t)-C\left(a^{2}+b^{2}\right) \\
& -2 a \int\left(v_{x} Q_{x}+v Q\right) \phi(t)-2 b \int\left(v_{x} Q_{x x}+v Q_{x}\right) \phi(t) .
\end{aligned}
$$

But

$$
\left|a \int\left(v_{x} Q_{x}+v Q\right) \phi(t)\right| \leq C a\left(\int\left(v_{x}^{2}+v^{2}\right) \phi(t)\right)^{1 / 2} \leq C^{2} a^{2}+\frac{1}{4} \int\left(v_{x}^{2}+v^{2}\right) \phi(t) .
$$

Doing the same for $\int\left(v_{x} Q_{x x}+v Q_{x}\right) \phi(t)$, we get

$$
\int\left(v_{1 x}^{2}+v_{1}^{2}\right) \phi(t) \geq \frac{1}{2} \int\left(v_{x}^{2}+v^{2}\right) \phi(t)-C\left(a^{2}+b^{2}\right),
$$

so that finally,

$$
H_{\phi}(v, v) \geq \lambda_{2} / 2 \int\left(v_{x}^{2}+v^{2}\right) \phi(t)-C e^{-\frac{\sigma_{0} \sqrt{\sigma 0}}{2} t}\|v\|_{H^{1}}^{2}-C\left(a^{2}+b^{2}\right) .
$$

Choosing $T_{1}$ so that $C e^{-\frac{\sigma_{0} \sqrt{\sigma_{0}}}{2}} T_{1} \leq \lambda_{2} / 4$, as $t \geq T_{1}$, this gives :

$$
H_{\phi}(v, v) \geq \lambda_{2} / 2 \int\left(v_{x}^{2}+v^{2}\right) \phi(t)-C\left(a^{2}+b^{2}\right) .
$$

\section{References}

[1] Jean Bourgain and Wensheng Wang, Construction of blowup solutions for the nonlinear Schrödinger equation with critical nonlinearity. Dedicated to Ennio De Giorgi., Annali della Scuola Normale Superiore di Pisa. Classe di Scienze. (4) 25 (1997), no. 1-2, 197-215.

[2] Raphaël Côte, Construction of solutions to the gKdV equations with a given asymptotic behaviour : the subcritical case, Preprint.

[3] L Large data wave operator for the generalized Korteweg-de Vries equations, to appear in Differential and Integral Equations 19 (2005), no. 2, 163-188. 
[4] Wiktor Eckhaus and Peter Schuur, The emergence of solutions of the Korteweg-de Vries equation form arbitrary initial conditions, Mathematical Methods in the Applied Sciences 5 (1983), 97-116.

[5] Nakao Hayashi and Pavel I. Naumkin, Large time asymptotic of solutions to the generalized Korteweg-De Vries equation, Journal of Functional Analysis 159 (1998), 110-136.

[6] Carlos E. Kenig, Gustavo Ponce, and Luis Vega, Well-posedness and scattering result for the generalized Korteweg-De Vries equation via contraction principle, Communications on Pure and Applied Mathematics 46 (1993), 527-620.

[7] - On the concentration of blow-up solutions for the generalized KdV equation critical in $l^{2}$, Contemporary Mathematics 263 (2000), 131-156.

[8] Diederik J. Korteweg and Gustav de Vries, On the change of form of long waves advancing in a rectangular canal, and on a new type of long stationnary waves, Philosophical Magazine 539 (1895), 422-443.

[9] Joachim Krieger and Wilhelm Schlag, Non-generic blow-up solutions for the critical focusing NLS in 1-d, Preprint.

[10] George L. Jr. Lamb, Element of soliton theory, John Wiley \& Sons, New York, 1980.

[11] Yvan Martel, Asymptotic N-soliton-like solutions of the subcritical and critical generalized Korteweg-de Vries equations, American Journal of Mathematics 127 (2005), no. 5, 1103-1140.

[12] Yvan Martel and Frank Merle, A Liouville theorem for the critical generalized Kortewegde Vries equation, Journal de Mathématiques Pures et Appliquées 79 (2000), 339-425.

[13] _ Asymptotic stability of solitons for subcritical generalized KdV equations., Archive for Rational Mechanics and Analysis 157 (2001), no. 3, 219-254.

[14] _ Instability of solitons for the critical generalized Korteweg-De Vries equation, Geometric and Functional Analysis 11 (2001), no. 1, 74-123.

[15] _ Blow up in finite time and dynamics of blow up solutions for the $L^{2}$-critical generalized KdV equations, Journal of the American Mathematical Society 15 (2002), $617-664$.

[16] _ Stability of blow-up profile and lower bounds for blow-up rate for the critical generalized kdv equation, Annals of Mathematics 1 (2002), 235-280.

[17] Yvan Martel, Frank Merle, and Tai-Peng Tsai, Stability and asymptotic stability in the energy space of the sum of $n$ solitons for subcritical gKdV equations, Communications in Mathematical Physics 231 (2002), 347-373.

[18] Frank Merle, Construction of solutions with exactly $k$ blow-up points for the Schrödinger equation with critical nonlinearity, Communications in Mathematical Physics 129 (1990), no. 2, 223-240.

[19] _ Existence of blow-up solutions in the energy space for the critical generalized Korteweg-de Vries equation, Journal of the American Mathematical Society 14 (2001), $555-578$.

[20] Robert M. Miura, The Korteweg-de Vries equation : a survey of results, SIAM Review 18 (1976), 412-459.

[21] Peter Schuur, Asymptotic analysis of solitons problems, vol. 1232, Springer-Verlag, Berlin, 1986.

[22] Panagiotis E. Souganidis and Walter A. Strauss, Instability of a class of dispersive solitary waves., Proceedings of the Royal Society of Edinburgh. Section A. Mathematics (1990), no. 3-4, 195-212.

[23] Michael I. Weinstein, Nonlinear schrödinger equations and sharp interpolations estimates., Communications in Mathematical Physics 87 (1983), no. 4, 567-576.

RAPHÄ̈L Côte

Département de Mathématiques et Applications, École normale supérieure,

45, rue d'Ulm, 75230 Paris Cedex 05, France

raphael.cote@ens.fr 